\title{
PERMISSIBLE CURVATURE OF PRISM SURFACES AND INACCURACY OF COLLIMATION IN PRECISE MINI- MUM-DEVIATION REFRACTOMETRY
}

\author{
By L. W. Tilton
}

\section{ABSTRACT}

Care in the optimum translational adjustment of a prism in order to permit the symmetrical use of all apertures is always necessary on account of aberrations inherent in lens systems. Such prism adjustments are advisable also because of slight curvatures of most prism surfaces. When prisms are at all times correctly located with respect to the axes of collimator, telescope, and spectrometer, the departures of the surfaces from planeness may then be appreciably greater than has hitherto been recognized as allowable. Moreover, it is shown that the latitude in collimation becomes sufficiently large to permit making all necessary refocusings with the telescope, even when using objectives with the usual type of color correction. Tolerances, corresponding to an error of $\pm 1 \times 10^{-6}$ in index of refraction, are evaluated for curvature of prism surfaces, translational adjustment of the prism, eccentricity of prism-table axis, and collimator refocusing.

\section{CONTENTS}

I. Introduction

II. Symmetrical use of the prism and the lens systems

1. Lens aberration and "obliquity" errors

2. Methods of refracting-angle measurement and curvature of prism surfaces

3. Minimum-deviation measurement and curvature of prism surfaces.

III. Relations between curvature of prism surfaces and prism-position adjustment.

1. Effect of asymmetric tabling when prism surfaces are curved (collimated incident light)

(a) Making refracting-angle measurements

(b) Making minimum-deviation measurements

(c) Combined effects of asymmetric tabling on refractiveindex determinations....

(d) Tolerance in curvature of prism surfaces

(e) Tolerance in asymmetric tabling

(f) Refocusing of telescope required because of prismsurface curvature.

2. Effect of eccentric prism-table axis when prism surfaces are curved (collimated incident light)

(a) Making refracting-angle measurements

(b) Making minimum-deviation measurements

(c) Combined effects of table-axis eccentricity on refractive-index determinations.

(d) Tolerance in table-axis eccentricity

IV. Relations between inaccurate collimation and prism-position adjustment_...............

1. Effect of asymmetric tabling when incident light is uncollimated (flat prism surfaces)

2. Effect of eccentric prism-table axis when incident light is uncollimated (flat prism surfaces)

(a) Experimental determination and azimuthal adjustment of eccentricity of table-axis

(b) Tolerance in collimation adjustment corresponding to a table-axis eccentricity of $0.2 \mathrm{~mm}$

V. Relation of prism aberration to collimation and to curvature of surfaces.

VI. Summary and discussion

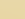




\section{INTRODUCTION}

The errors of a goniometrical nature which occur in the practice of precise prism refractometry may be classed as pertaining either $(a)$ to the spectrometer only and to its use as a goniometer, or $(b)$ to the prism and its relation to the instrument. The most important errors of the first category are those pertaining to the divided circle and in the second group the proper orientation of a prism in azimuth has often been considered a serious matter. Both of these subjects have been discussed by the author in former papers. ${ }^{1}$ In this paper consideration is given chiefly to other interrelations of prism and spectrometer, and the stipulations which are customarily made regarding the planeness of prism surfaces are discussed. In particular, the accuracy necessary in the translational adjustment of the prism with respect to the instrument is considered.

The adjustments of the spectrometer itself are in general well understood, but they require particular mention in two instances. The customarily assumed necessity for securing exact collimation has not been conclusively demonstrated and, on the other hand, it is not apparent that the eccentricity of the prism-table axis can be safely neglected under all the conditions which occur in practice. Both collimation and axis eccentricity are, however, closely related to prism quality and to translations of the prism, and consequently these two adjustments of the spectrometer are discussed in this paper because they cannot be adequately considered apart from a treatment of other matters relating more particularly to the prism.

Although planeness of prism surface is the only prism quality explicitly considered, a high degree of homogeneity is necessarily assumed, especially in treating of the subject of prism aberration and its bearing on the permissible inaccuracies of collimation. It is also presupposed that the working conditions are such that the properties of the prism are satisfactorily constant during the measurements; and many other obvious matters are not mentioned.

In all cases the individual tolerances are here evaluated to corresspond to an error of $\pm 1 \times 10^{-6}$ in index of refraction. While these tolerances are given as consistent with "sixth decimal place" refractometry, it should be remembered that, in order to limit the combined errors rigorously to one unit of the sixth place, the separate contributions must be confined to still smaller magnitudes.

For convenience of reference the definitions of the various symbols are summarized here as follows:

$A \equiv$ refracting angle of an isosceles portion of a prism (measured between planes tangent at the mid-points of the effective prism surfaces);

$A_{e} \equiv$ erroneous value of refracting angle as measured when prism is incorrectly placed on the prism table;

$C$ and $C^{\prime} \equiv$ intersections of incident and emergent chief rays for prism in the "left-hand" position and in the "right-hand" position, respectively $\left(C^{\prime}\right.$ sometimes coinciding with $C$ );

\footnotetext{
${ }^{1}$ L. W. Tilton, B.S.Jour. Research, vol. 2 (RP64), p. 909, 1929; vol. 6 (RP262), p. 59, 1931.
} 
$c$ and $c^{\prime} \equiv$ cosines of incidence angles on first and second faces of prism as oriented for minimum deviation; that is,

$$
c \equiv \cos \frac{A+D}{2} \text { and } c^{\prime} \equiv \cos (A / 2) ;
$$

$D, D_{e}$, and $D_{\epsilon} \equiv$ angles of minimum deviation produced, respectively, by a prism correctly placed, asymmetrically tabled, and tabled with respect to an eccentric table axis;

$E \equiv_{\lambda}$ distance through which, after refractingangle measurement, the prism vertex should be translated toward the table axis preparatory to deviation measurement for a wave length $\lambda$;

$e_{A} \equiv$ linear asymmetry of prism position (when making refracting-angle measurements) measured from prism-table axis to the intersection of normals erected at the surface centers of the effective isosceles portion of the prism;

$e_{D} \equiv$ linear asymmetry of prism position (when making minimum-deviation measurements) measured from prism-table axis to the intersection of the incident and emergent chief rays;

$e_{A R}$ and $e_{A L} \equiv$ errors in tabling a prism for refractingangle measurement as measured perpendicularly to the line of sight at the right- and left-hand telescope pointings;

$e_{D R}$ and $e_{D L} \equiv$ errors in tabling a prism for minimumdeviation measurement as measured perpendicularly to the line of sight at the right- and left-hand pointings;

$e_{m} \equiv$ maximum error (measured perpendicularly to the telescope pointings) that is made in translational adjustment of a prism;

$\epsilon \equiv$ linear eccentricity of prism-table axis with respect to axis of the spectrometer;

$\eta \equiv$ phase difference in complete periods or cycles;

$f \equiv$ focal length of telescope objective;

$f_{c} \equiv$ focal length of collimator objective;

$f^{\prime}{ }_{c} \equiv$ adequate collimator focal length which permits refractive-index measurement without chromatic refocusing of collimator;

$f_{t p} \equiv$ combined focal length of telescope objective and prism;

$F_{c} \equiv$ actual collimator tube length or distaxice from collimator slit to objective; 
$\Delta F_{c} \equiv$ error in focusing collimator; that is, $\Delta F_{c}=\left(f_{c}-F_{c}\right)$;

$\Delta F_{A} \equiv$ required refocusing of telescope for refracting-angle measurements (on curved-surface prisms) by auto-collimation;

$\Delta F_{D} \equiv$ required refocusing of telescope for minimum-deviation measurements; that is, $\Delta F_{D}=\left(f_{t p}-f\right)$

$\varphi \equiv$ azimuth of eccentricity of prism-table axis;

$L \equiv$ length or horizontal dimension of an effective prism face or surface;

$\lambda \equiv$ wave length of light;

$n \equiv$ relative index of refraction of a medium, the subscripts $C$ and $F$ specifying the wave lengths 6,563 and $4,861 \mathrm{~A}$, respectively;

$n^{\prime} \equiv$ Rayleigh's symbol for $n c^{\prime} / c$;

$\delta n_{\triangle A}$ and $\delta n_{\Delta D} \equiv$ partial errors in index of refraction attributable to error in measurements of $A$ and $D$, respectively;

$\Delta n_{e}, \Delta n_{\epsilon}$, and $\Delta n_{\Delta F_{c}} \equiv$ combined errors in index of refraction attributable to asymemtric tabling, eccentricity of prism-table axis, and inaccurate collimation, respectively;

$N_{A}$ and $N_{D} \equiv$ number of measurements of refracting: angle and minimum deviation, respectively, made with independent translational adjustments of the prism;

$\nu \equiv$ optical constringence, that is, $\nu=\frac{n-1}{n_{2}-n_{1}}$;

$O \equiv$ horizontal projection of the vertical axis. of the spectrometer;

P.E. $e_{0} \equiv$ probable error of a single translational adjustment of a prism made perpendicularly to the telescope axis;

P.E. $n_{A}$ and P.E. $n_{D} \equiv$ partial probable errors in index of refraction attributable to probable error of tabling as it affects measurements of $A$ and $D$, respectively;

P.E. $n_{e} \equiv$ combined probable error in index of refraction attributable to probable errors in asymmetry of tabling the prism;

$r_{1}$ and $r_{2} \equiv$ radii of curvature of faces of a prism (positive when convex toward incident light);

$r \equiv$ an average of the radii of curvature of faces of a prism; that is, $r=0.5\left(r_{1}-r_{2}\right)$;

$S \equiv$ position of source;

$S_{1}$ and $S_{2} \equiv$ virtual positions of source (for left- and right-hand pointings, respectively) when prism is properly tabled and table-axis. eccentricity is zero; 
$S_{L}$ and $S_{R} \equiv$ virtual positions of source (for left- and right-hand pointings) when prism is not in correct position with respect to the spectrometer axis;

$s \equiv$ sagittal departure (in wave lengths) from flatness for any prism surface, $s^{\prime}$ referring to a limited portion of the surface having a diameter of $1 \mathrm{~cm}$;

$T_{1 / r} \equiv$ tolerance in average prism-surface curvature which corresponds to a probable error of $\pm 1 \times 10^{-6}$ in refractive index;

$T_{\text {P.E. } e_{0}} \equiv$ tolerance in probable error of translational adjustment of a prism (made perpendicularly to the telescope axis) which corresponds to a probable error of $\pm 1 \times 10^{-6}$ in refractive index;

$T_{\epsilon} \equiv$ tolerance in table-axis eccentricity which corresponds to an error of $\pm 1 \times 10^{-6}$ in refractive index;

$T_{\Delta F_{c}} \equiv$ tolerance in inaccuracy of collimation which corresponds to an error of $\pm 1 \times 10^{-6}$ in refractive index;

$\vartheta_{A}$ and $\vartheta_{D} \equiv$ azimuth of asymmetry of prism position (for refracting-angle and minimum-deviation measurement, respectively) referred to the bisector of the refracting angle (positive toward base of prism); $u$ and $u^{\prime} \equiv$ object and image distances measured from first surface of lens (or prism); $v \equiv$ image distance measured from second surface of lens (or prism);

$x \equiv$ distance from collimator objective to prism;

$y \equiv$ semiwidth of (cross-sectional) aperture of the pencil incident on the prism; and

$y^{\prime} \equiv$ projection of semidiameter of incident pencil along the first face of the prism; that is, $y^{\prime} \equiv y / c$.

\section{SYMMETRICAL USE OF THE PRISM AND THE LENS SYSTEMS}

The necessity of proper translational adjustment of the prism with respect to the axis of the spectrometer has been noticed to some extent by several writers. Hastings, ${ }^{2}$ originally, exercised considerable care in tabling his prisms in order to eliminate errors due to aberration which he recognized as existing in every objective, and later he made further statements about the intersection of lines of collimation and prism-face centers. Müller ${ }^{3}$ had both curvature of prism surfaces and lens aberration in mind when speaking of prism positions on the table, at least when referring to measurements of refracting angles. Macé de Lépinay ${ }^{4}$ refers to errors in focusing the collimator and to the

2 C. S. Hastings, Am. J. Sci., vol. 15, pp. 269-275, 1878; vol. 35, pp. 65-68, 1888.

3 G. Müller, Publicationen des Astrophysikalischen Observatoriums zu Potsdam, $\nabla$ ol. 4, p. $163,1885$.

4 J. Macé de Lépinay, J. de Physique (2), vol. 6, pp. 190-196, 1887. See, also, Annales de Chimie et de Physique (7), vol. 5, pp. 225-226, 1895. 
curvatures of surfaces as reasons for adjusting the prism so that when measuring deviations the chief ray of the beam of incident light passes through the centers of the prism surfaces. It seems, however, that the majority of observers have given inadequate attention to these matters, and some recent manuals, in treating of refractive index goniometry, have neglected them entirely. ${ }^{5}$ Moreover no one seems to have realized that strictly symmetrical conditions permit the use of curved surfaces on prisms and a useful latitude in collimation. Therefore, on account of the advantages in observing the principle of symmetry, and also because of its fundamental importance for good work, even in the fifth decimal place of refractive index, it is well to discuss this topic in detail.

\section{LENS ABERRATION AND "OBLIQUITY" ERRORS}

In general, comparatively small prisms and large telescope apertures are used in prism refractometry, and it is especially under these conditions that the greatest care must be taken to make the effective apertures symmetrical about vertical lines through the lens and prism-surface ${ }^{6}$ centers. Otherwise, the presence of aberration in the lens system, a departure from flatness of the prism surfaces, or an unusually defective collimator adjustment may vitiate the results.

Furthermore, even with perfect lenses, flat prism surfaces, and perfect adjustment of collimator tube length, an error in pointing is still introduced, according to Guild, ${ }^{7}$ by inaccurate focusing of the eyepiece, whenever oblique cones of rays are produced within the telescope through using the objectives ${ }^{8}$ unsymmetrically. This causes an "obliquity" error in angular measurement unless the asymmetry is of a compensating nature at each of the two pointings.

\section{METHODS OF REFRACTING-ANGLE MEASUREMENT AND CURVA- TURE OF PRISM SURFACES}

The difficulties in connection with the unsymmetrical use of apertures and with oblique reflections from imperfect prism surfaces are, in fact, so great that the split-beam method of measuring a refracting angle, $A$, is probably inadequate in precise refractometry even with the application of troublesome corrections, such as those given by Cornu ${ }^{9}$ or Carvallo ${ }^{10}$ for (1) the absolute error in collimation, and (2) the changes in focus necessitated by the curvatures of prism surfaces; and this statement is made with due consideration of the apparent advantage of the procedure in that $2 A$ is directly determined, thus halving certain errors in $A$.

With any method of refracting-angle measurement, the curvature of prism surfaces must be regular to give fair imagery by reflection. Curvatures must also be of the same character (both convex or both

\footnotetext{
3 The Dictionary of A pplied Physics, vol. 4, Macmillan \& Co., Ltd., London, is a noteworthy exception and for this credit is due to J. Guild, of the National Physical Laboratory.

6 If large prisms are used and the whole telescopic apertures flled, then for curved prism surfaces, it is still necessary to use prism apertures which are symmetrical about definite vertical lines, conveniently those through the surface centers.

7 J. Guild, Proc., Phys. Soc., London, vol. 28, p. 244, 1916; or Nat. Phys. Lab., Collected Researches, vol. 13, p. 232, 1916. See also W. Uhink, Zeits. 1. Instrumentenk,. vol. 52, pp. 435-442, 1932.

8 Obviously, the unsymmetrical use of an eyepiece causes no errors because both image and fiducial lines are equally displaced.

A. Cornu, Annales de l'Ecole Normale Superieure (2), vol. 9, pp. 76-87, 1880.

10 E. Carvallo, Annales de l'Ecole Normale Superieure (3), vol. 7, supplement, pp. 77-88, 1890.
} 
concave) and approximately ${ }^{11}$ of the same magnitude to obviate refocusing of the telescope between two successive pointings. When a prism satisfies these conditions and is leveled and "centered" so that the face-center normals intersect the vertical axis of a properly adjusted spectrometer, the prim angle measured by autocollimation (and possibly by collimator and rotating table ${ }^{12}$ also) will be sensibly that between planes tangent at the mid-points of the prism surfaces. This value of the prism angle is, however, not the refracting angle actually used at minimum deviation except for isosceles prisms. Only an isosceles portion of a prism can be used for minimum deviations and the refracting angle $A$, as considered in this paper, is formed by planes tangent at the mid-points of the effective surfaces of the isosceles portion. The circumcenter of the horizontal projection of this usable portion of the prism must coincide with the spectrometer axis during refracting-angle measurements. ${ }^{13}$

\section{MINIMUM-DEVIATION MEASUREMENT AND CURVATURE OF PRISM SURFACES}

If, after refracting-angle measurement, the prism be properly translated so that, when measuring minimum deviation, the axes of the telescope and collimator again intersect the effective prism surfaces at their mid-points, then the measured deviation will correspond very closely ${ }^{14}$ to that for a plane-surface prism of the refracting angle determined by the tangent planes at these mid-points. For a prism with curved surfaces this particular plane-surface-prism deviation is defined as the correct value.

For other prism positions, which may be termed asymmetric (axis of telescope or collimator not intersecting effective prism-surface center) different measured values of refracting angle and of minimum deviation are to be expected, depending on the amount of asymmetry of position, the curvature of the surfaces, and perhaps on the lack of collimation. It is, of course, realized that these variations in the measured values depend, also, on certain differential errors (lens aberration and obliquity) due to variations in the unsymmetrical use of the optical system. However, with small asymmetries of prism position (see figs. 4 and 6), and fairly well corrected objectives, it may be considered that these differential errors are negligible ${ }^{15}$ in comparison with the primary effects which are to be discussed. Also, as will be found in section $\mathrm{V}$, it can be assumed that the aberration of the prism is likewise of minor importance. From this standpoint the result of asymmetric prism position will now be considered in detail, first in connection with curvature of surfaces, section III, and then in its relation to lack of collimation, section IV. For convenience and simplicity of treatment, asymmetry of prism position will be considered

\footnotetext{
11 See footnote 17, p. 32 , and footnote 27, p. 42 .

12 Although the lens system is used unsymmetrically in the prism-rotation method of angle measurement with a collimator, it will be noticed that the asymmetry can (for isosceles prisms) be identical for the successive pointings. The reflections at oblique incidence are, however, unfavorable because of aberration introduced by imperfect prism surfaces. W. Voigt (Zeits. f. Kryst., vol. 5, pp. 122-124, 1880) has discussed a special case of the error caused by incorrect translational adjustment when measuring by this method the refracting angle of a prism having curved surfaces.

${ }_{13}$ See footnote 20, p. 34 .

14 References to prism-aberration errors are made in section $V$.

15 In all cases which are considered in detail in this paper, these neglected differential errors pertaining to thelens systems may, if necessary, be eliminated by the use of centrical (preferably rectangular) diaphragms of adjustable aperture. The first order errors caused by using asymmetric apertures of prisms having curved surfaces are, of course, not obviated by the use of such diaphragms.
} 
as arising from two separate causes (1) asymmetry of tabling; that is, failure to correctly translate and adjust the prism with reference to the axis of the prism table; and (2) that incorrectness of prism position which may result solely because of eccentricity of the prism table axis.

\section{RELATIONS BETWEEN CURVATURE OF PRISM SUR- FACES AND PRISM-POSITION ADJUSTMENT}

In this section it will be assumed (1) that the spectrometer is equipped with perfectly corrected objectives (see footnote 15), (2) that the aberration introduced by the prism is negligibly small (see sec. V), (3) that the instrument and the prism are correctly adjusted in the usually mentioned particulars, including the accurate adjustment of the slit in the focal plane of the collimator objective, and (4) that the optical axes of both telescope and collimator intersect the principal axis of rotation of the instrument. ${ }^{16}$

With these assumptions the light incident on the prism when measuring deviations is strictly parallel and, if the prism surfaces are plane, the observing telescope may be used as correctly focused for infinity. Under these ideal conditions no particular care is required in translational adjustments when tabling prisms and no errors ensue from eccentricity of the prism table axis. It is quite otherwise, however, when the prism surfaces are curved, and the effects of asymmetric tabling and of eccentric table axis will be considered separately, with the additional general assumption (5) that the prism surfaces are both convex, or concave, and have radii which are approximately equal ${ }^{17}$ and very large compared to the dimensions of the prism.

\section{EFFECT OF ASYMMETRIC TABLING WHEN PRISM SURFACES ARE CURVED (COLLIMATED INCIDENT LIGHT)}

In discussing asymmetric tabling and prism-surface curvature it will further be specifically assumed $\left(6^{\prime}\right)$ that the asymmetry is small compared with the prism and that the prism-table axis is not only parallel to but coincides with that of the spectrometer.

\section{(a) MAKING REFRACTING-ANGLE MEASUREMENTS}

Referring first to refracting-angle measurement, attention will be confined to the autocollimation method ${ }^{18}$ with rotating telescope, and in figure 1 the axis of the prism table coincides with the axis of the spectrometer at $O$, while the intersection of the face-center normals of the isosceles portion of the prism is at $C$. The fiducial mark in the image plane of the telescope is replaced by its virtual positions at $S_{L}$ and $S_{R}$, the centers of curvature of the prism surfaces, and $S_{L} t_{L}$ and $\mathrm{S}_{R} t_{R}$ are normals to the prism surfaces at their centers.

\footnotetext{
${ }^{16}$ In this directional adjustment of a telescope or a collimator no elaborate attempt need be made to use, in practice, a true optical axis but merely the line from the image plane fiducial mark, or from the slit center, approximately through the appropriate principal point of the objective. These lines may vary somewhat in azimuth as the tube lengths are changed and so do not exactly intersect the vertical axis of the spectrometer except, possibly, for one particular tube length. All pointings are thus to be regarded as slightly erroneous, but no direct effect of this remains in the resultant angles provided the tube lengths remain constant between pointings. (Slight inaccuracies in prism-position adjustment may result.) The introduction of assumption (4) serves, however, to simplify the discussion in this and in the following section.

17 The radii must be equal only to the extent that during refracting-angle measurement a satisfactory compromise focus of the telescope can be found. Excellence of definition is not of great importance because the precision of tabling (see fig. $4(b)$ ) insures approximate symmetry of the aberration about a vertical axis. See footnote 27, p. 42 .

${ }_{18}$ The general assumption (3) of collimated incident light must, of course, be interpreted here as "autocollimated" light for the particular surfaces concerned.
} 
The refracting angle (in radians) whose measure is required is

$$
A=\pi-\angle t_{L} C t_{R}
$$

but since the telescope revolves about $O$, it takes the directions $T_{L} O S_{L}$ and $T_{R} O S_{R}$, irrespective of the tube length or of the obliquity of the eccentric pencils, and consequently

$$
A_{e}=\pi-\angle T_{L} O T_{R}
$$

is the angle which is determined. From equations (1) and (2), and from the figure, it is evident that

$$
\Delta A=\angle t_{L} S_{L} T_{L}+\angle T_{R} S_{R} t_{R}
$$

is the error in angle, namely, $A_{e}-A$.

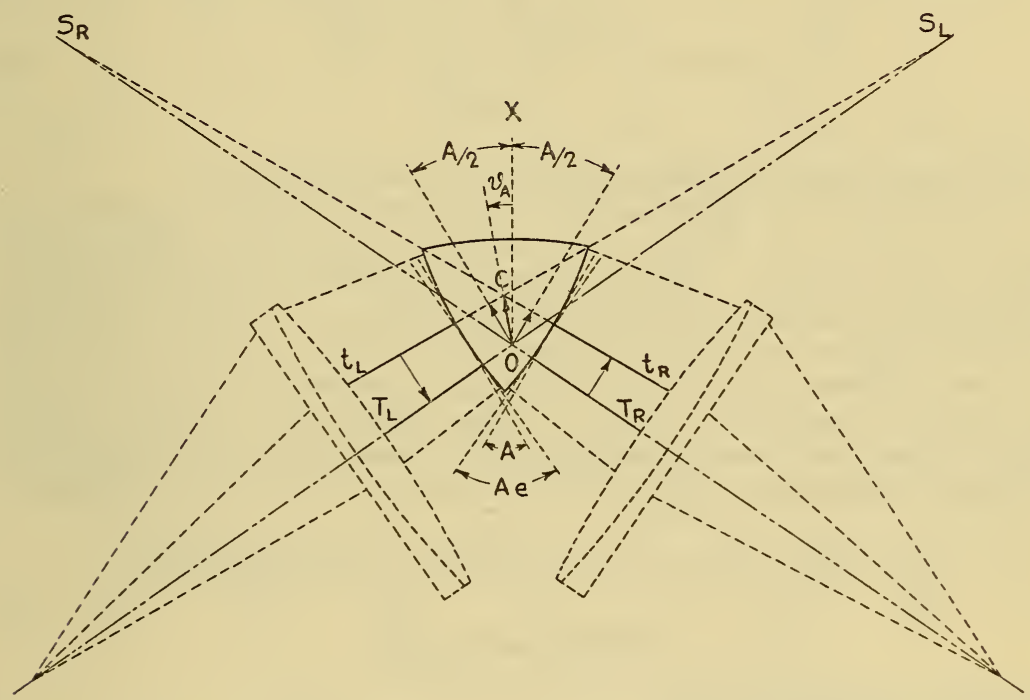

FIGURE 1.-Autocollimation measurement of the refracting angle of an asymmetrically tabled prism having curved surfaces.

When the intersection of the face-conter normals, $t_{L} C S_{L}$ and $t_{R} C S_{R}$, at the prism "center" $C$, is not coincident with the vertical axis of the goniometer at $O$, then the telescope pointings which are necessarily along $T_{L} O$ and $T_{R} O$ toward the virtual sources $S_{L}$ and $S_{R}$ are not parallel to the normals. Thus the chief error in determining the definite refracting angle $A$ is directly proportional to the curvature of the prism surfaces and also to $O C \cos \theta_{A}$ which is the longitudinal component of the error in tabling. Of the other errors which occur because of the unsymmetrical use of the telescope objectives, some are independent of prism surface curvature but all may be minimized by reducing the asymmetry of tabling.

If from $O$, parallel to the bisector of the refracting angle of the prism, a reference line, $O X$, is drawn, the positive direction being toward the third side of the prism, then $O C$, the asymmetry of prism position, may conveniently be considered as a vector having a length $e_{A}$ and an azimuth $\vartheta_{A}$ with respect to this reference line. The lengths of the components of $O C$, perpendicular to $t_{L} C$ and $t_{R} C$, respectively, are $e_{A} \cos \left(A / 2-\vartheta_{A}\right)$ and $e_{A} \cos \left(A / 2+\vartheta_{A}\right)$ where $A / 2$ is an essentially positive quantity. Consequently equation (3) may be easily rewritten ${ }^{19}$ as

${ }_{10}$ To rewrite equation (4) in seconds of arc apply the factor $206.3 \times 10^{3}$. 


$$
\Delta A=\frac{2 e_{A} \cos \frac{A}{2} \cos \vartheta_{A}}{r}
$$

where $r=0.5\left(r_{1}-r_{2}\right)$ is an average radius of curvature, positive for convex surfaces. Then by the use of the appropriate elemental differential equation for the minimum-deviation method, namely

$$
\frac{o n}{o A}=-\frac{\sin \frac{D}{2}}{2 \sin ^{2} \frac{A}{2}}
$$

one obtains

$$
\delta n_{\Delta A}=\frac{-e_{A} \sin \frac{D}{2} \cos \vartheta_{A} \cos \frac{A}{2}}{r \sin ^{2} \frac{A}{2}}
$$

as the partial effect of prism surface curvature on index.

A consideration of equation (4) and the conditions of its derivation shows that for a given refracting angle this error is independent of prism-table rotation. Thus equation (4) applies not only to "direct" measurements of any refracting angle, but also to "reverse" determinations of the same angle by measurements on its explement. If, however, all three angles of any prism are measured for a given tabling adjustment, ${ }^{20}$ the prism table remaining stationary or being conveniently rotated between measurements on each angle, the asymmetry of tabling affects each angle differently but from their sum the error

$$
\begin{aligned}
\Delta A_{1}+\Delta A_{2}+\Delta A_{3}= & \frac{2 e_{A}}{r}\left(\cos \frac{A_{1}}{2} \cos \vartheta_{A 1}+\cos \frac{A_{2}}{2} \cos \vartheta_{A 2}\right. \\
& \left.+\cos \frac{A_{3}}{2} \cos \vartheta_{A 3}\right)
\end{aligned}
$$

vanishes completely since

$$
\vartheta_{A 2}=\vartheta_{A 1}+\pi+\frac{A_{1}+A_{2}}{2} \text { and } \vartheta_{A 3}=\vartheta_{A 2}-\pi+\frac{A_{2}+A_{3}}{2},
$$

and by a series of trigonometric transformations it can be shown that the total factor in parentheses equals zero. This result, which is quite obvious from geometrical considerations, is of value when making a precise test of a goniometer with a prism polished on all three faces.

(b) MAKING MINIMUM-DEVIATION MEASUREMENTS

As already mentioned, the prism-face-center normals should not intersect the table axis during deviation measurements. The refracting edge or vertex of an isosceles portion of the prism, as placed for angle measurement, should be moved toward the table axis a distance

$$
E \lambda=\frac{L}{2} \tan \frac{A}{2} \sec \frac{D}{2} \sin \frac{A+D}{2}
$$

${ }^{20}$ In general it is the circumcenter of the horizontal projection of the entire prism which must coincide with the vertical axis of the spectrometer during prism-angle measurements. Only for equilateral prisms are all three of these prism angles equivalent to refracting angles for index measurement. See sec. II, 2. 
$L$ being the length of a face of the isosceles prism. The axes of telescope and collimator then always pierce the centers of the effective surfaces when the minimum-deviation conditions are fulfilled and thus this correct installation of the prism may also be termed symmetrical. Equation (8) is equivalent to that given by Carvallo, ${ }^{21}$ who also demonstrated that, in passing from deviation on the left to that on the right, the conditions of symmetry are preserved without further prism translation.

In practice, when more than one wave length is used for refractive index measurements, it is advisable to know whether or not a prism requires retabling between the various deviation measurements.

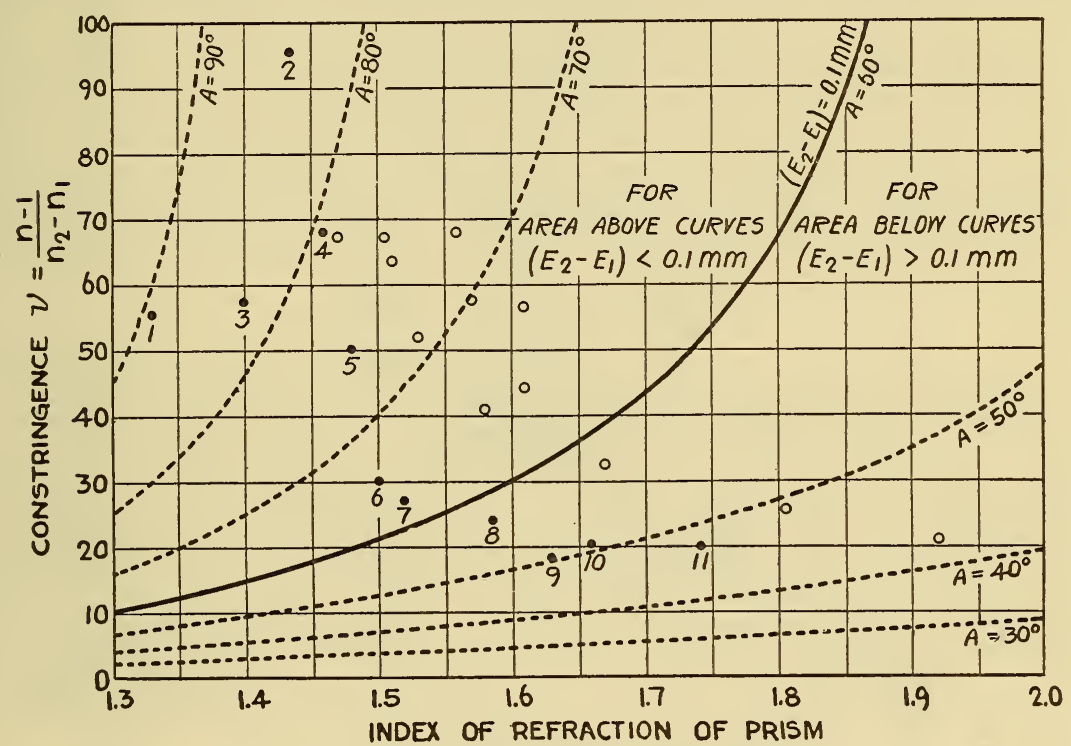

FiguRE 2.-Chromatic tolerance in retabling.

These contours of $\left(E_{2}-E_{1}\right)=0.1 \mathrm{~mm}$ show, for various refracting angles, the chromatic limits for a given translational prism adjustment for minimum-deviation measurements. A prism surface length of $2 \mathrm{~cm}$ is used and a precision of $\pm 0.1 \mathrm{~mm}$ in prism translation is assumed. If, for any spectral interval $\left(\lambda_{2}-\lambda_{1}\right)$, the corresponding constringence of a substance lies above the $\Delta E$ curve for the appropriate $A$, then no retabling is advisable between observations on spectral lines separated by a comparable interval. For the particular interval $\left(n_{F}-n_{C}\right)$, the open circles designate typical optical glasses and the dots show approximate locations of other substances as follows: 1 , water; 2 , fluorite; $3, n$-octane; 4 , fused quartz; 5 , linseed oil 6 , benzene; 7 , tungoil; 8 , aniline; 9 , carbon disulphide; $10, m$-bromonaphthalene; 11 , methylene iodide.

Accordingly, equation (8) has been used in computing these chromatic variations. A prism-surface length of $2 \mathrm{~cm}$ has been used and results are expressed in figure 2 for several values of prism angle. The ordinate $\nu$ is a general expression (see fig. 2) inversely proportional to the partial dispersion between any two wave lengths. For the sodium lines index and the special spectrum interval from 4,861 to $6,563 \mathrm{~A}$ ( $F$ to $C$ of hydrogen) this becomes that particular measure of optical constringence which was introduced by Ernst Abbé and is now widely used for expressing dispersion data. For this spectrum interval the locations of several transparent media are shown by circles and dots on figure 2. In using this figure it should be remembered that (1) prisms in excess of approximately $2 \mathrm{~cm}$ surface length are seldom if

${ }^{21}$ See pp. 89-92 of paper cited in footnote 10, p. 30 . 
ever required for index measurements; ${ }^{22}$ (2) the attainable precision of a single adjustment of prism position is $P . E .= \pm 0.1 \mathrm{~mm}$ or less (see sec. III, 1 (c) below); (3) as shown by figure 4 a precision as high as P.E. $= \pm 0.1 \mathrm{~mm}$ in this prism adjustment is seldom necessary; and (4) the partial dispersion $\left(n_{F}-n_{C}\right)$ is, for most transparent media, approximately one half the partial dispersion for the whole range of the visible spectrum. Consequently, it may be concluded that comparatively few prisms, of angle $A=60^{\circ}$ or less, require any translational readjustment on the prism table during dispersion measure-

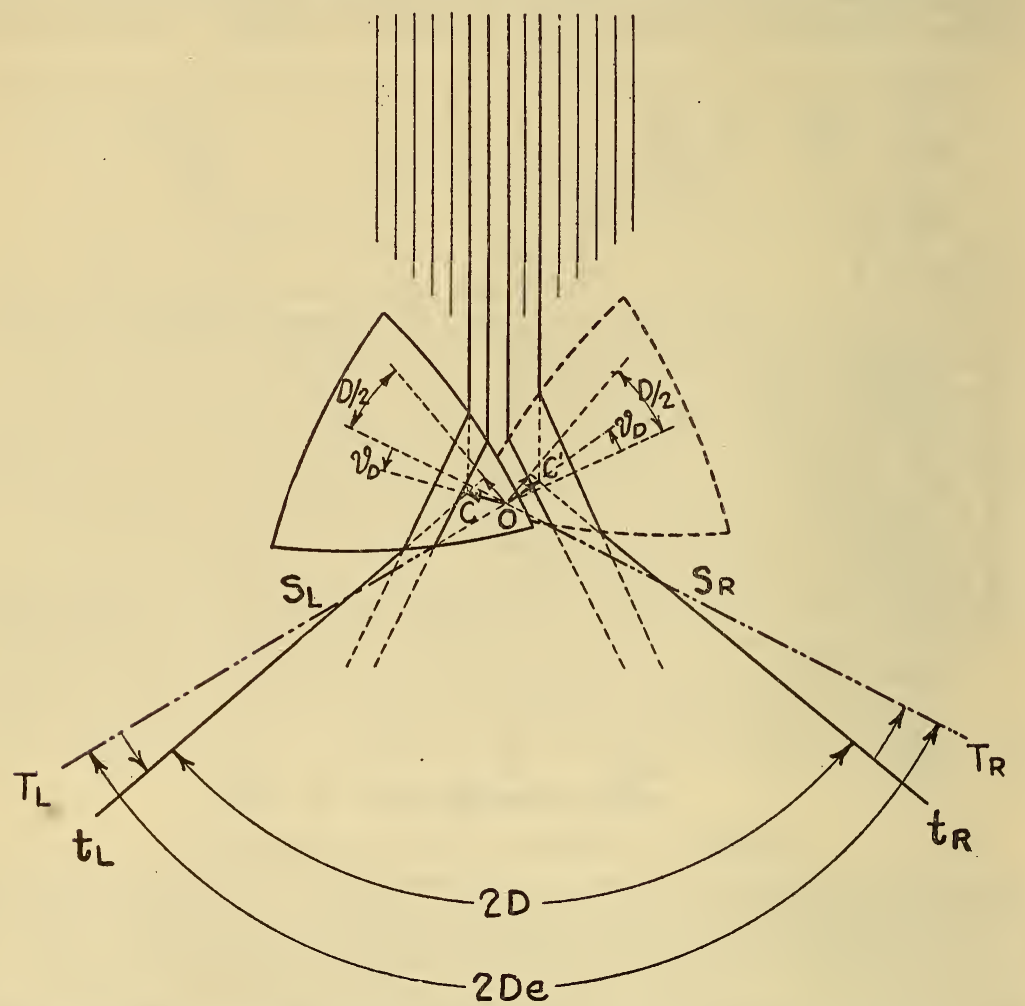

FIGURE 3.-Minimum-deviation measurement of an asymmetrically tabled prism having curved surfaces.

Images of the source are formed at $S_{L}$ and $S_{R}$ when the prism is oriented for deviation left and right, respectively. $C$ and $C^{\prime}$ are the intersections of the prolongations of those rays which traverse the prism surface centers and in correct tabling for deviation measurement these points must coincide at $O$. The emergent rays $C S_{L} t_{L}$ and $C^{\prime} S_{R} t_{R}$ have the angular separation $2 D$, the measurement of which is desired, but the telescope pointings are necessarily along $T_{L} S_{L}$ and $T_{R} S_{R}$ toward the virtual sources and the axis of the spectrometer at $O$.

ments within the range of the visible spectrum, if the initial adjustment is made for some wave length near the midrange.

To facilitate the investigation of errors in minimum-deviation measurements which result from incorrect or asymmetric prism tabling, reference will be made to figure 3 where the coincident axes are again represented at $O$ while $C$ and $C^{\prime}$ are the points of intersection of those particular incident and emergent chief rays which pass through the surface centers during deviation left and deviation right, 
respectively. The collimator is supposed replaced by a source at an infinite distance, $u$, and at the corresponding image distance, $v$, from the prism is located the virtual source, $S_{L}$, to be imaged in the telescope at the left-hand pointing. A similar virtual source, $S_{R}$, is shown for the right-hand pointing.

Obviously, for a given orientation, only a single ray traverses the prism exactly at minimum deviation but the extreme divergence of the emergent rays with which one is concerned is usually only a matter of seconds. Consequently it is evident ${ }^{23}$ that one prism orientation serves sufficiently well for all such rays.

If the observing telescope measured correctly the deviation of the ray which passes through the prism-surface centers, that is one half the angle, $2 D$, between $t_{L} C$ and the similar line $t_{R} C^{\prime}$, then no error would be caused by the curvature of surfaces because these particular rays traverse the prism precisely as if the surfaces were flat and thus (with collimated incident light) the effective lateral translation of the prism as it is oriented about $O$ between left and right deviation would be to this extent immaterial. The telescope, however, swings through the angle $T_{L} O T_{R}=2 D_{e}$ between left and right pointings, and therefore

$$
2 \Delta D=\angle T_{L} S_{L} t_{L}+\angle t_{R} S_{R} T_{R}
$$

expresses the error in double deviation.

With the same convention previously used regarding the azimuth, $\vartheta_{D}$, of the asymmetry $O C=O C^{\prime}$, this distance may be resolved into components of length $e_{D} \cos \left(D / 2+\vartheta_{D}\right)$ and $e_{D} \cos \left(D / 2-\vartheta_{D}\right)$, perpendicular respectively to $t_{L} C$ and $t_{R} C^{\prime}$, where $D / 2$ is considered essentially positive. Neglecting prism thickness and the distance from the prism to the objective, the virtual object distance for telescope pointings is $O S_{L}=O S_{R}$, or approximately the image distance, $v$, as given by the formula for oblique refraction (primary plane) through a thin lens in air, namely

$$
\frac{1}{v}=\frac{1}{u}+\frac{n c^{\prime}-c}{c^{2}}\left(\frac{1}{r_{1}}-\frac{1}{r_{2}}\right)
$$

where in this application $u$ is infinite, $n$ is the index of refraction of the prism, $c$ and $c^{\prime}$ (cosines of incidence angles) are $\cos \frac{A+D}{2}$ and $\cos (A / 2)$, respectively, and $r_{2}=-r_{1}$ approximately. Consequently

$$
v=\frac{r}{2} \cdot \frac{\sin \frac{A}{2} \cos ^{2} \frac{A+D}{2}}{\sin \frac{D}{2}}
$$

and equation (9) becomes

$$
\Delta D=\frac{2 e_{D} \sin \frac{D}{2} \cos \vartheta_{D} \cos \frac{D}{2}}{r \sin \frac{A}{2} \cos ^{2} \frac{A+D}{2}}
$$

where, again, $r$ is positive for convex surfaces.

${ }^{23}$ For tolerance in prism orientation see fig. 5 , p. 73 of second paper cited in footnote 1, p. 26 . 
Using the value of $v$ expressed in equation (11) as the focal length of the prism at minimum deviation, the required refocusing of the telescope, of focal length $f$, is

$$
\Delta F_{D}=f_{t p}-f=\frac{-2 f^{2} \sin \frac{D}{2}}{r \sin \frac{A}{2} \cos ^{2} \frac{A+D}{2}+2 f \sin \frac{D}{2}}
$$

where $f_{t p}$ is the combined focal length of telescope objective and prism, and separation has been neglected. Consequently, equation (12) may be written as

$$
\Delta D=\frac{-e_{D} \Delta F_{D}}{f^{2}} \cos \vartheta_{D} \cos \frac{D}{2}
$$

if the relatively unimportant second term in the denominator of (13) is neglected. For the case of convex surfaces and the special conditions, $\vartheta_{D}=\pi$ and $e_{D}=E$ as expressed in equation (8), it is found that equation (14) is equivalent to that given by Carvallo ${ }^{24}$ as

$$
\Delta D=-\frac{p}{f^{2}} \delta f \sin \frac{A+D}{2}
$$

where $p$ is the perpendicular from the center to a side of the prism, and $\delta f=-\Delta F_{D}$.

By combining equation (12) with the appropriate elemental equation

$$
\frac{o n}{o D}=\frac{n}{2 \tan \frac{A+D}{2}}
$$

one obtains

$$
\delta n_{\Delta D}=\frac{e_{D} \sin \frac{D}{2} \cos \vartheta_{D} \cos \frac{D}{2}}{r \sin ^{2} \frac{A}{2} \cos \frac{A+D}{2}}
$$

as another partial effect of prism-surface curvature on index.

(c) COMBINED EFFECTS OF ASYMMETRIC TABLING ON REFRACTIVE-INDEX

Equations (6) and (17) may be added to give

$$
\Delta n_{e}=\frac{\sin \frac{D}{2}}{r \sin ^{2} \frac{A}{2}}\left(\frac{e_{D} \cos \vartheta_{D} \cos \frac{D}{2}}{\cos \frac{A+D}{2}}-e_{A} \cos \vartheta_{A} \cos \frac{A}{2}\right)
$$

as the total index error which occurs because of asymmetric tabling of prisms having curved surfaces.

For a prism having a given angle, refractive index, and curvature of surfaces, the sign and the magnitude of the error expressed by equation (18) are, of course, dependent on the lengths $e_{A} \cos \vartheta_{A}$ and $e_{D} \cos \vartheta_{D}$. In particular, it may be remarked that, under the assump-

${ }^{24}$ See p. 81 of paper cited in footnote 10, p. 30. 
tions which have been made in this section, translational prism adjustment at right angles to the bisector of the refracting angle (that is, at an azimuth $\vartheta= \pm \frac{\pi}{2}$ ) has no effect ${ }^{25}$ on measurements of refractive index.

If translational adjustments are made by centering the image of the prism in the exit pupil of the telescope while the latter is correctly pointed for measurements, then from diagrams similar to figures 1 and 3 it can easily be shown that

and

$$
e_{A} \cos \vartheta_{A}=\frac{e_{A R}+e_{A L}}{2} \sec \frac{A}{2}
$$

$$
\left.e_{D} \cos \vartheta_{D}=\frac{e_{D R}+e_{D L}}{2} \sec \frac{D}{2}\right\}
$$

where $e_{A R}, e_{A L}, e_{D R}$, and $e_{D L}$ are the tabling errors, measured perpendicularly to the line of sight, which are made at each of the right- and left-hand pointings required in angle and in deviation measurements. All four of these centerings in the line of sight can be made with approximately the same precision and, if $e_{m}$ is the maximum error to be made, then equation (18) can be rewritten as

$$
\Delta n_{e}= \pm \frac{e_{m} \sin \frac{D}{2}}{r \sin ^{2} \frac{A}{2}}\left(\sec \frac{A+D}{2}+1\right)
$$

to express the limiting errors in index for the most unfavorable case which may occur.

Next, a typical value for $e_{m}$ is required. Obviously, some care must be taken if this error is to be small, say within the limits of $\pm 1 \mathrm{~mm}$, and if the precision sought is to correspond to a valid accuracy. The author mounts vertical threads near the centers of the objectives so that they intersect those effective axes of the telescope and collimator which are properly directed (see assumption (4) and footnote 16 of sec. III), and he uses a prism table provided with two sets of horizontal ways which are operated by slow-motion screws. Then, while viewing a magnified image of the exit pupil of the telescope, one may center any prism aperture (less than 2 or $3 \mathrm{~cm}$ in width) with a probable error not exceeding $\pm 0.08 \mathrm{~mm}$ for a single translational adjustment. Consequently, a limiting value for $e_{m}$ may be taken as approximately $\pm 0.4 \mathrm{~mm}$ and for a $60^{\circ}$ prism of index 1.5 it may be found from equation (20) that $r$ must be as large as $1,280 \mathrm{~m}$ to obviate index error greater than $\pm 1 \times 10^{-6}$.

Similar calculations for special cases with even larger estimated values for $e_{m}$ are probably responsible in part for the widely prevalent idea that extremely flat prism surfaces are absolutely essential for accurate refractive-index measurements. Fortunately, such a large error as $e_{m}$ is to be expected but once in a thousand of such prism adjustments, and, moreover, as shown by equation (18), there are ${ }^{25}$ It should be remembered, however, that in practice a neglect of lateral translational adjustment leads
to appreciable aberrational and obliquity errors (see sec. II, 1). 
two terms which have opposite signs and will, in the great majority of cases, compensate to some appreciable degree. Thus probability has an important bearing on the practical matter of establishing a tolerance in curvature for prism surfaces. Therefore it is suggested that a useful tolerance in this curvature should correspond to the production of a given probable error in index through measurements on a prism so adjusted that the probable error of each of the four independent single translations (made perpendicularly to the telescope axis) is P.E.e.

Corresponding to the actual errors of equation (19), the probable errors are

$$
\left.\begin{array}{l}
P . E \cdot e_{A} \cos \vartheta_{A}= \pm \frac{P \cdot E \cdot e_{0}}{\sqrt{2}} \sec \frac{A}{2} \\
P . E \cdot e_{D} \cos \vartheta_{D}= \pm \frac{P \cdot E \cdot e_{0}}{\sqrt{2}} \sec \frac{D}{2}
\end{array}\right\}
$$

and these may be substituted for actual errors in equations (6) and (17) to express the separate probable errors in index, P.E. $n_{A}$ and P.E. $n_{D}$, which result from probable errors in prism tabling during single refracting-angle and deviation measurements, respectively. P.E. $n_{A}$ and P.E. $n_{D}$ may then be combined as

$$
P . E . n_{e}= \pm \frac{P . E \cdot e_{0}}{\sqrt{2}} \frac{\sin \frac{D}{2}}{r \sin ^{2} \frac{A}{2}} \sqrt{1+\sec ^{2} \frac{A+D}{2}}
$$

to express the effect of tabling probable error in a single complete refractive-index determination instead of the actual error of equation (18) or the maximum error of equation (20).

If $N_{A}$ measurements of angle and $N_{D}$ measurements of deviation are made with readjustments of prism position, the quantity under the radical sign of equation (22) becomes

$$
\frac{1}{N_{A}}+\frac{1}{N_{D}} \sec ^{2} \frac{A+D}{2}
$$

and obviously it is advantageous to choose $N_{A}$ and $N_{D}$ so that approximate equality of these two terms is obtained. Thus, for any desired precision in index measurements to be made on a given prism with a definite available degree of tabling precision, one may easily decide to what extent the prism tabling should be repeated during such repetitions as may for other reasons be included on the observational program.

Equation (22) also serves as a suitable basis for determining (1) tolerances in curvature for prism surfaces which are to be centered or tabled with a given precision, and (2) tolerances in imprecision of tabling prisms having given curvature of surfaces. 
(d) TOLERANCE IN CURVATURE OF PRISM SURFACES

From equation (22) the tolerance in curvature is

$$
T_{1 / r}= \pm \frac{1.41 \times 10^{-6} \sin ^{2} \frac{A}{2}}{P . E \cdot e_{0} \sin \frac{D}{2} \sqrt{1+\sec ^{2} \frac{A+D}{2}}}
$$

if the error in index is specified as P.E. $n^{\prime}{ }_{e}= \pm 1 \times 10^{-6}$ for a single determination of refractivity. Equation (23) has been used in computing reference contours which are shown in figure $4(a)$ and labeled

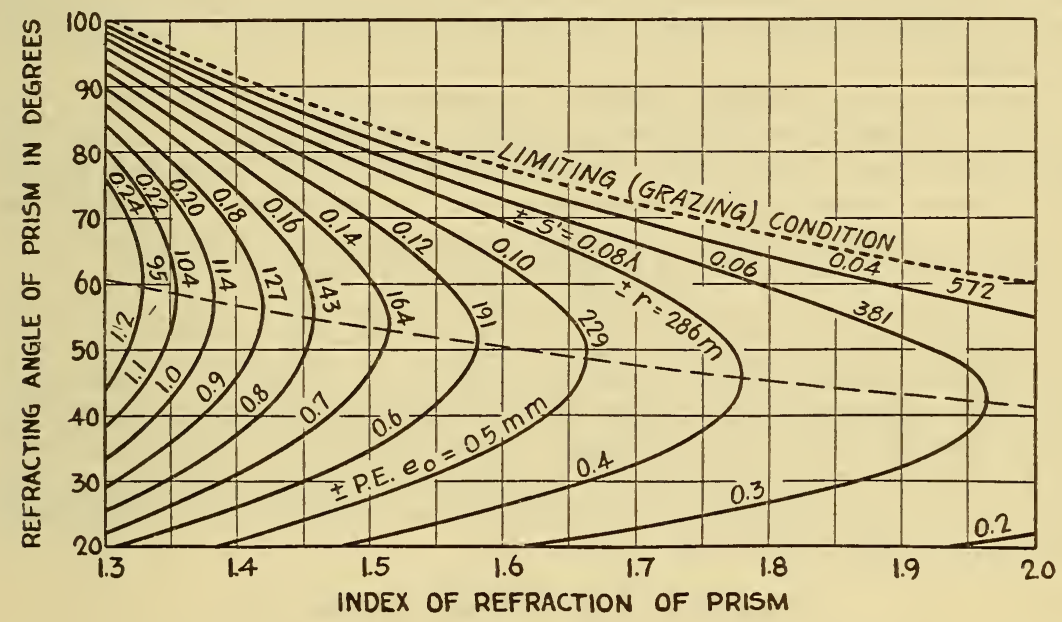

Figure 4

(a) Tolerance contours for approximately equicurvature of prism surfaces. - These contours of permissible curvature (read designations on upper arms of curves) are computed for translational adjustments of a prism which are made with a precision $P . E . e^{\prime} 0 \equiv \pm 0.1 \mathrm{~mm}$. Departures from flatness are expressed as radius of curvature in meters and also as the sagitta, $s^{\prime}$, between the curved surface and a plane which passes through the circumference of a surface area having a diameter, $d^{\prime} \equiv 1 \mathrm{~cm}$. If the curvatures are those specified, an idex of refraction may be measured with a precision $P . E \cdot n^{\prime}{ }^{\prime} \equiv \pm 1 \times 10^{-6}$.

(b) Tolerance contours for precision of prism translation. If the prism surfaces depart from flatness by only $0.02 \lambda$ for an area of $1 \mathrm{~cm}$ diameter $(r= \pm 1,145 \mathrm{~m})$ then these same curves show (as designated on their lower arms) the precision necessary in the installation of a prism on the spectrometer table when an index precise to $\pm 1 \times 10^{-6}$ (probable error) is to be measured.

in the upper portion of the diagram. ${ }^{26}$ The particular value P.E. $e^{\prime}{ }_{0}=$ $\pm 0.1 \mathrm{~mm}$, used in these computations, was selected because it is a unit value corresponding closely to a precision reached experimentally with the use of simple auxiliary prism-tabling devices. Since the planeness of prism surfaces is conveniently tested by comparison with a standard flat surface, the tolerances have been expressed as departures, $s^{\prime}$, in wave lengths $(\lambda=0.546 \mu)$ from flatness for a surface having a diameter, $d^{\prime}$, of $1 \mathrm{~cm}$; that is, $s^{\prime}$ represents departures of the curved surfaces from a plane tangent to the central point of this

${ }^{26} \mathrm{By}$ taking with respect to $A$ the partial derivative of the tolerance in curvature (equation 23 expressed in terms of the variables $A$ and $n$ ), the most favorable conditions is found to be

$$
\tan \left(A+\frac{D}{2}\right) \tan ^{3} \frac{A+D}{2}=2
$$

and this is expressed in fig. 4 by an undesignated dashed line. 
limited area. The general relation between the sagitta, $s$, the diameter, $d$, and radius of curvature, $r$, is $s=d^{2} / 8 r$, an expression which, in connection with equation (22), shows that the limiting permissible sagitta for any area of surface varies directly as $d^{2}$ and directly as $P$. E. $n_{e}$, the permissable probable error in index, but varies inversely as P.E.e. .

Tolerances or precisions may thus be readily evaluated from figure 4 (a) for any given conditions according to the equation

$$
\frac{P \cdot E \cdot n_{e}}{P \cdot E \cdot n^{\prime}{ }_{e}}=\frac{P \cdot E \cdot e_{0}}{P \cdot E \cdot e^{\prime}} \cdot \frac{s}{s^{\prime}} \cdot \frac{d^{\prime 2}}{d^{2}}
$$

where unprimed symbols refer to actual conditions for any given case and the primes denote the specific conditions for which figure $4(a)$ is drawn.

For example, if a $60^{\circ}$ prism of fluorite, index 1.434 , with $2 \mathrm{~cm}$ surfaces, is being polished for an index determination to $\pm 1 \times 10^{-6}$ (probable error), the surfaces are satisfactory if they show like departures from planeness not exceeding $0.7 \lambda$, provided a precision of P.E. $e_{0}= \pm 0.1 \mathrm{~mm}$ can be realized in translational adjustment of the prism. Or, if P.E. $e_{0}= \pm 0.2 \mathrm{~mm}$ and a $50^{\circ}$ prism of index $n=1.9$ has surfaces of $2.5 \mathrm{~cm}$ in length which have like curvatures corresponding to an over-all sagitta of $2 \lambda$ each $(|r|=74 \mathrm{~m})$, the index may nevertheless be measured correctly to $P . E .= \pm 1 \times 10^{-5}$. In practice, however, serious difficulty may be encountered with the larger values of permissible curvature because in such cases two surfaces of the same prism frequently differ too much ${ }^{27}$ in the degree of their curvature to permit a satisfactory compromise focusing of the telescope when measuring refracting angles.

(e) TOLERANCE IN ASYMMETRIC TABLING

From equation (22) the tolerance in imprecision of tabling is

$$
T_{P . E . e_{o}}= \pm \frac{1.41 \times 10^{-6} \sin ^{2} \frac{A}{2}}{\frac{1}{r} \sin \frac{D}{2} \sqrt{1+\sec ^{2} \frac{A+D}{2}}}
$$

if the probable error in index is to be $\pm 1 \times 10^{-6}$ for a single determination of refractivity. Equation (24) has been used in computing reference curves which coincide with those shown in figure $4(a)$ but, in this case, they are redesignated in the lower portion of the diagram and considered as figure $4(b)$. The choosing of the curvature for these computations was somewhat arbitrary. The value of $\underline{l} r \underline{l}=$ $1,145 \mathrm{~m}$ which is used corresponds to a departure from flatness of $1 / 50$ wave length $(\lambda=0.546 \mu)$ at the circumference of an area having a diameter of $1 \mathrm{~cm}$ (or $1 / 8 \lambda$ for an area 1 inch in diameter) and is a fairly high degree of planeness even for precise optical surfaces of small prisms. The curves of figure $4(b)$ thus show approximately the maximum freedom in probable error of translational prism adjustment which is consistent with probable errors of unity in the sixth decimal place of refractive index. Evidently precision of prism

${ }_{27}$ For a $400-\mathrm{mm}$ collimator the permissible difference in radii may, however, be at least as large as $0.3 r$ when $|r|=100 \mathrm{~m}$ and as large as $1.0 r$ when (average) $|r|=500 \mathrm{~m}$. See footnote $17, \mathrm{p} .32$ 
installation to fractions of a millimeter should invariably be obtainable, the necessary precision being higher for larger curvatures and relatively much higher for media of high index than for those of low index. Incidentally, it may also be mentioned that correct tabling to small fractions of a millimeter is desirable from a purely goniometrical viewpoint, as shown by equation (4), if accurate or even precise measurements are to be made on angles between (planes tangent to) curved surfaces.

Since the probable errors in index, according to equation (22), are directly proportional to those probable errors which may be made in tabling, it becomes evident from figure 4 that the customary total neglect of prism position may cause serious error even in the fifth decimal place of indices for prisms having only very slight surface curvature. It is principally because of this required high precision in tabling, and the consequent enforced symmetrical use of all optical surfaces, that it seems at all permissible, as suggested in section II, to neglect the consideration of tolerances for the accuracy of eyepiece focusing and for residual aberrations in fairly well corrected optical systems.

\section{(f) REFOCUSING OF TELESCOPE REQUIRED BECAUSE OF PRISM-SURFACE CURVATURE}

In connection with the discussion of collimation, to be considered in sections IV and V, it is of interest to determine the maximum refocusing of the telescope which is required on account of the use of prisms with curved surfaces. For minimum-deviation measurements equation (13) serves, and for the refocusing in angle measurement by autocollimation, $\Delta F_{A}$, the similar equation is

$$
\Delta F_{A}=\frac{f^{2}}{r-(f-z)}
$$

where $z$ is the distance from a prism surface to the telescope objective. The quantity $(f-z)$ is negligible in comparison with $r$ and if the latter be taken as positive for convex reflecting surfaces the refocusing is positive for an increase in telescope tube length. From the formulas (13) and (25), it may be ascertained that with $f=400 \mathrm{~mm}$ the range of curvatures shown in figure $4(a)$ will necessitate telescope refocusings of from 0.3 to $1.7 \mathrm{~mm}$ when making refracting-angle measurements and from 2.5 to $6.5 \mathrm{~mm}$ for deviation observations on $60^{\circ}$ prisms ranging from 1.3 to 1.9 in index. These refocusings are of the same order of magnitude as those which are found in section IV, $2(b)$ as permissible changes in collimator tube length. It is not apparent, however, that these required changes in the telescope tube length can produce any further errors comparable with those which are discussed in this section as effects of the curvature of prism surfaces.

\section{EFFECT OF ECCENTRIC PRISM-TABLE AXIS WHEN PRISM SUR- FACES ARE CURVED (COLLIMATED INCIDENT LIGHT)}

In treating of the eccentricity of prism-table axis and of its relation to the measurement of prisms having curved surfaces, the special assumption $\left(6^{\prime}\right)$ of part 1 of this section is no longer valid because the table axis in this case is not coincident with that of the instrument but merely parallel thereto as covered by general assumption (2). Instead it will now be assumed $\left(6^{\prime \prime}\right)$ that the table-axis eccentricity 
is small and that the prism is correctly placed ${ }^{28}$ with respect to the axis of the table.

\section{(a) MAKING REFRACTING-ANGLE MEASUREMENTS}

When "directly" measuring a refracting angle (by autocolimation) with a rotating telescope, eccentricity of table axis has no significance as distinguished from asymmetry of table position of the prism because no table rotation is involved. The corresponding error in index may, therefore, be determined at once by comparing with equation (6) and adopting a new reference system. If linear eccentricity of table axis is denoted by $\epsilon$, and $\varphi$ represents its azimuth with respect to the (fixed) collimator axis, the positive direction being that of the incident light, then the partial error in index, namely, that part due to erroneous refracting-angle measurement, is expressible as

$$
\delta n_{\Delta_{A}}=\frac{\epsilon \sin \frac{D}{2} \cos (\varphi-\sigma) \cos \frac{A}{2}}{r \sin ^{2} \frac{A}{2}}
$$

where $\sigma$ is the angle through which the prism is oriented from the customary symmetrical installation in which the bisector of $A$ makes an angle of $180^{\circ}$ with the collimator axis during refracting-angle measurements. Consequently it is possible without knowing $\epsilon$ or $\varphi$ to eliminate eccentric table-axis error from the average of "direct" and "reverse" measurements on $(\pi-A)$ and $(\pi+A)$, respectively, by choosing for these two prism installations two values of $\sigma$ which differ by $\pi$. Also, it should be noted that, in case $\varphi$ is known but cannot be satisfactorily adjusted (see $2(a)$ of sec. IV), $\sigma$ may often be chosen in such manner as to eliminate or effectually minimize table-axis error in "direct" goniometry of prisms having curved surfaces.

Obviously, if the collimator does not prevent the telescope from making a complete revolution, the three angles of a prism may be "directly" measured without table rotation and their sum obtained free from eccentric table-axis error just as for the analogous case of asymmetric tabling expressed by equation (7). When, however, the prism table is rotated between prism-angle measurements so that in each case the bisector of $A$ and the collimator axis form an angle of $180^{\circ}$, then (see equation (4)) the sum of the three angles exceeds $180^{\circ}$ by the amount

$$
\Delta \Sigma=\left\{\xi-\frac{412.5 \times 10^{3} \epsilon \cos \varphi}{r}\left(\cos \frac{A_{1}}{2}+\cos \frac{A_{2}}{2}+\cos \frac{A_{3}}{2}\right)\right\} \text { sec. }
$$

where $\xi$ is the excess in seconds due to the pyramidal error of the prism and the remaining term must also be considered when seeking to accurately check the performance of a goniometer by applying this test to "direct" measurements.

${ }^{28}$ That is, "centered" over the table axis during refracting-angle measurements (see sec. II, 2) and then displaced according to equation (8) for deviation measurements. 
(b) MAKING MINIMUM-DEVIATION MEASUREMENTS

In order to derive an expression for the error in minimum-deviation measurement when the prism-table axis is eccentric, figure 5 has been drawn. The principal difference between this case and the one represented in figure 3 is that here $O$ represents only the axis of the instrument and for both right- and left-hand deviations the eccentric axis of the prism table now coincides at $C=C^{\prime}$ with the intersection of those rays which traverse the prism surface centers. As previously, the correct angular deviation is one half the angle between $t_{L} C$ and $t_{R} C$, although the telescope swings through the angle $T_{L} O T_{R}$. Therefore

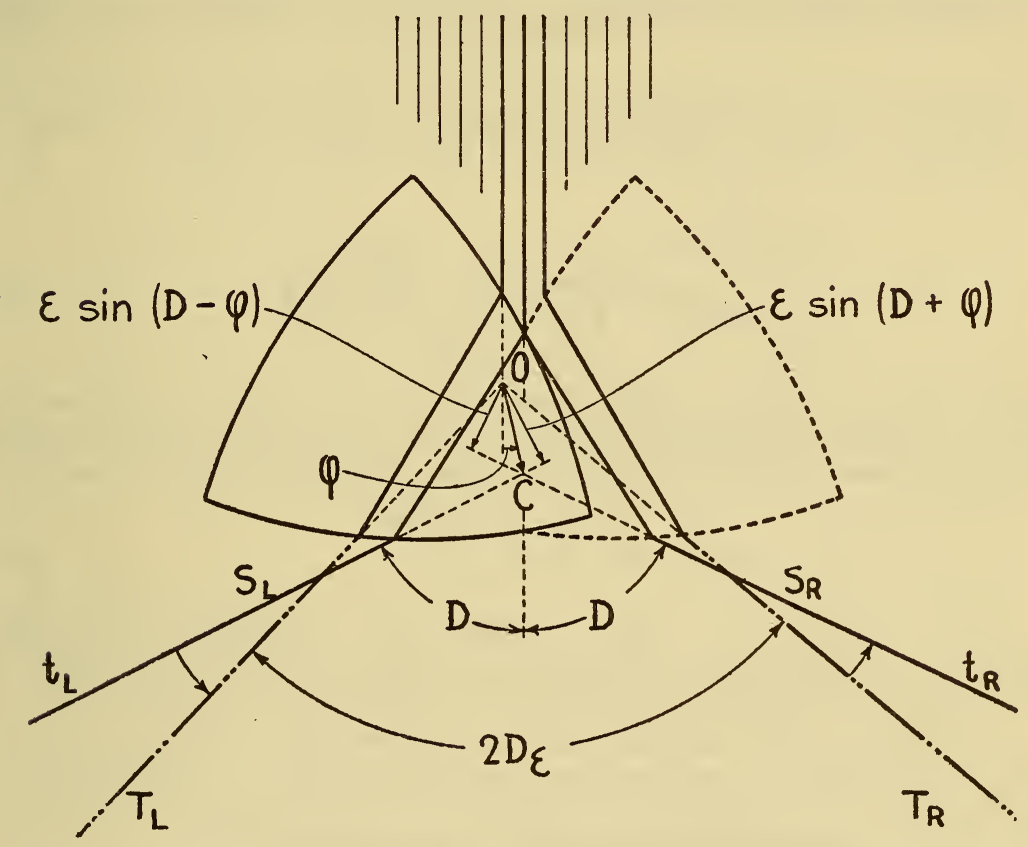

FIGURE 5.-Minimum-deviation measurement of a prism symmetrically tabled with respect to an eccentric prism-table axis when the prism surfaces are curved

The incident and emergent chief ray intersections, shown on flgure 3 at $C$ and $C^{\prime}$, are here coincident with the eccentric prism-table axis at $C$. The telescope pointings include the angle $2 D_{\epsilon}$ instead of $2 D$ which would be measured if the table axis and the spectrometer axis were coincident at $O$.

$$
2 \Delta D=-\angle t_{L} S_{L} T_{L}-\angle T_{R} S_{R} t_{R}
$$

is the error in double deviation.

According to the convention adopted for expressing the azimuth of the eccentricity of the table axis, $\varphi$ in figure 5 is a positive angle in the first quadrant, and it is evident that $O C$ may be resolved into the components $\epsilon \sin (D+\varphi)$ and $\epsilon \sin (D-\varphi)$, perpendicular, respectively, to $t_{L} C$ and $t_{R} C$, where $D$ is considered as essentially positive. Consequently, using equations (11) and (28), it is found that

$$
\Delta D=\frac{-2 \epsilon \sin \frac{D}{2} \cos \varphi \sin D}{r \sin \frac{A}{2} \cos ^{2} \frac{A+D}{2}}
$$


and then from equation (16) one obtains

$$
\delta n_{\Delta D}=\frac{-\epsilon \sin \frac{D}{2} \cos \varphi \sin D}{r \sin ^{2} \frac{A}{2} \cos \frac{A+D}{2}}
$$

as the partial error in index resulting from that error in minimumdeviation measurement which arises because of curved prism surfaces and eccentricity of prism-table axis.

(c) COMBINED EFFECTS OF TABLE-AXIS ECCENTRICITY ON REFRACTIVE-INDEX

Usually in refracting-angle measurements $\sigma$ is zero and the value of $\epsilon \cos \varphi$ is; of course, identical in equations (26) and (30). Then by the addition of these partial errors in index, there results

$$
\Delta n_{\epsilon}=\frac{\epsilon \sin \frac{D}{2} \cos \varphi}{r \sin ^{2} \frac{A}{2}}\left(\cos \frac{A}{2}-\frac{\sin D}{\cos \frac{A+D}{2}}\right)
$$

which is an expression for the total error in index due to any eccentricity of table axis which may exist when measuring prisms having curved surfaces. This error becomes zero for the particular condition

$$
\sin D=\cos \frac{A}{2} \cos \frac{A+D}{2}
$$

and also whenever the value of $\varphi= \pm 90^{\circ}$. The latter possibility indicates an important and general method of eliminating errors of this nature, provided that $\varphi$ is known and that the prism table support may itself be rotated with respect to the base of the instrument. In part 2 of section IV a method for the determination of $\varphi$ and $\epsilon$ will be mentioned.

(d) TOLERANCE IN TABLE-AXIS ECCENTRICITY

From equation (31) a tolerance in table-axis eccentricity is

$$
T_{\epsilon}= \pm \frac{1 \times 10^{-6} \sin ^{2} \frac{A}{2}}{\frac{1}{r} \sin \frac{D}{2} \cos \varphi} \cdot \frac{\cos \frac{A+D}{2}}{\cos \frac{A}{2} \cos \frac{A+D}{2}-\sin D}
$$

for an error of $\pm 1 \times 10^{-6}$ in index measurements on prisms having surface curvature $1 / r$. Figure 6 gives an idea of the extent to which unfavorably oriented table-axis eccentricity $\left(\varphi=0\right.$ or $\left.180^{\circ}\right)$ may be tolerated, according to equation (33), when using prisms whose surfaces have precisely as much curvature as may for other reasons (see fig. $4(a)$ ) be permitted. In other words, that variable value of $1 / r$ which is defined by equation (23) has been used in solving equation (33) for figure 6. 
The degree of permissible table-axis eccentricity, for refractiveindex determinations, thus proves to be satisfactorily large in general and especially so for $60^{\circ}$ prisms in the range 1.4 to 1.6 of refractive index. This is mainly the result of compensating effects of errors in refracting-angle measurement and of those made in observations of minimum deviation. Such liberal tolerances in table-axis eccentricity are, however, by no means desirable for all other purposes. A wellbuilt goniometer for precise work should not have table-axis eccentricity in excess of 0.1 or $0.2 \mathrm{~mm}$ because, for example, with $\epsilon=0.2 \mathrm{~mm}$ and $r=572 \mathrm{~m}(s=0.04 \lambda$ for $1 \mathrm{~cm})$, the error in the sum of the measured angles of a $60^{\circ}$ prism, according to equation (27), may be as large as 0.4 seconds unless one uses averages of measurements made both on the angles and on their explements.

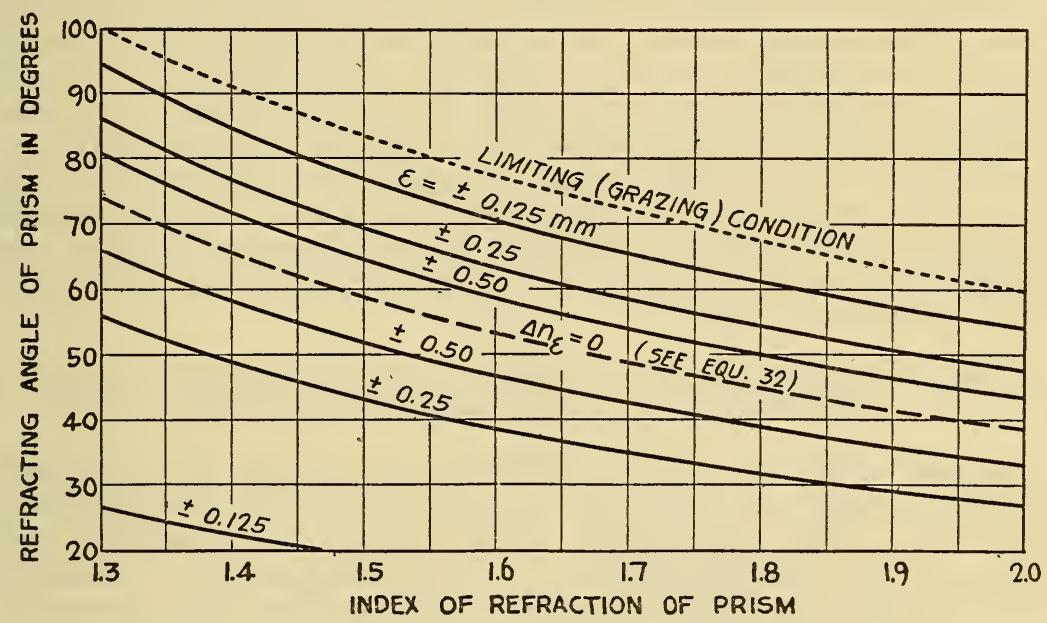

FIGURE 6.-Tolerance contours for prism-table-axis eccentricity

If prism surfaces have no equicurvature in excess of the values shown in figure $4(a)$, then table-axis eccentricity is permissible to the extents shown here even when its azimuth is 0 or $180^{\circ}$ with respect to the collimator axis. These tclerances apply only to complete refractive-index determinations. For accurate goniometry of prisms having only slight curvature of surfaces this eccentricity should not exceed $0.2 \mathrm{~mm}$.

\section{RELATIONS BETWEEN INACCURATE COLLIMATION AND PRISMI-POSITION ADJUSTMENT}

Parallel light over a prism table is probably never realized not only on account of the difficulty in securing, except by chance, a perfect optimum adjustment of collimator tube length for the existing conditions of a particular moment but also because of aberration inherent in the lens system. This collimation adjustment of a spectrometer has been considered of such importance that ordinary care has been deemed inadequate, presumably on account of depth of focus, and special procedures have been recommended, such, for example, as Schuster's ${ }^{29}$ method of alternately refocusing the telescope and the collimator on appropriately produced slit images, or the refocusing in pairs ${ }^{30}$ of three telescopic systems of which one is the collimator in question.

${ }^{20}$ Schuster, Phil. Mag. (5), vol. 7, p. 95, 1879.

30 See p. 765 of volume cited in footnote 5, p. 29. $176983-33-4$ 
Possibly current ideas on the necessity of equal refocusing of both collimator and telescope are traceable to Cornu ${ }^{31}$ and Carvallo, ${ }^{32}$ who showed that, when measuring angles by the split-beam method, the corrections for curvature of prism surfaces were eliminated by refocusing both tubes to equal extents. Considerable attention may also have been directed to precise collimation because imperfections of this nature were considered by Macé de Lépinay and Buisson as having caused the discrepancies between certain spectrometrically and interferometrically determined indices. ${ }^{33}$

It seems quite necessary then to investigate the effects of erroneous collimation of the incident light. Only minimum-deviation measurements, however, need consideration because the autocollimating method of refracting-angle measurement (which, as indicated in sec. II, is preferable for work of highest accuracy) is automatically eliminated from the discussion. As a simplification, the general assumtions of the previous section, namely, assumptions (1) to (5), inclusive, will be used here, except that (3) must obviously be modified to include merely an approximate rather than an accurate collimation adjustment, so that there exists an error in collimator focusing, $\Delta F_{c}=f_{c}-F_{c}$, where $F_{c}$ is the actual collimator tube length (from slit to objective) and $f_{c}$ is the focal length of the collimator objective; also assumption (5) will be reduced to the special case $r=\infty$, or that the prism surfaces are plane.

\section{EFFECT OF ASYMMETRIC TABLING WHEN INCIDENT LIGHT IS UNCOLLIMATED (FLAT PRISM SURFACES)}

Assumption $\left(6^{\prime}\right)$ of part 1 , section IV, concerning the coincidence of axes, will again be used here, and in figure 7 these axes are represented at $O$. The intersection of the incident and emergent chief rays is at $C$ for deviation left and at $C^{\prime}$ for deviation right. A distant source, $S$, replaces the collimator; $S_{1}$ and $S_{2}$ are the virtual sources for pointings when a prism is properly centered at $O$, and the correct double deviation is $2 D=\angle T_{1} O T_{2}$. Points at $S_{L}$ and $S_{R}$ represent the virtual sources when the tabling is asymmetric. The paths in air of the rays for left and right deviations then lie on lines from $S$ to $C$ to $t_{L}$ and $S$ to $C^{\prime}$ to $t_{R}$, respectively, and the measured double deviation is $2 D_{e}=\angle T_{L} O T_{R}$. The additional angle designations

and

$$
\left.\begin{array}{l}
\alpha_{1}=\angle O S C^{\prime} \\
\beta_{1}=\angle C S O \\
\alpha_{2}=\angle T_{R} S_{R} t_{R} \\
\beta_{2}=\angle t_{L} S_{L} T_{L} \\
2 d=\angle t_{L} S_{L} \text { to } t_{R} S_{R}
\end{array}\right\}
$$

will be employed for convenience. Then, from figure 7

$$
2 d=2 D_{e}+\alpha_{2}+\beta_{2}
$$

31 See p. 87 of paper cited in footnote 9, p. 30 .

32 See pp. 85-88 of paper cited in footnote 10, p. 30 .

33 For brief remarks and references see p. 915 of first paper cited in footnote 1, p. 26 . 
and, since $\alpha_{1}+\beta_{1}$ is the effective change of orientation in the incident light between left and right pointings,

$$
2 d=2 D+\alpha_{1}+\beta_{1}
$$

whence

$$
\Delta D=\frac{\alpha_{1}-\alpha_{2}+\beta_{1}-\beta_{2}}{2}
$$

may be written to express the error in measured deviation.

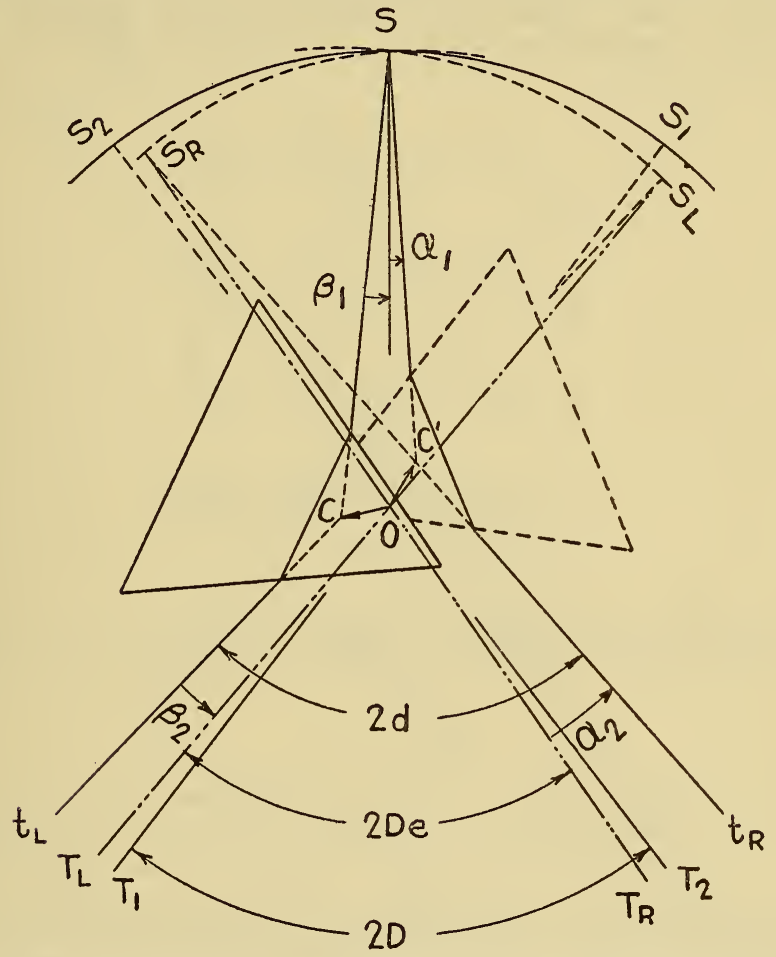

FIGURE 7.-Minimum-deviation measurement of an asymmetrically-tabled prism when using uncollimated incident light

Although the telescope pointings are along $T_{L} O$ and $T_{R} O$ toward the virtual sources $S_{L}$ and $S_{R}$, investigation shows that the (double) minimum deviation thereby measured is equal to that for a symmetrically tabled prism (not shown) for which $C$ and $C^{\prime}$ would coincide at $O$, the axis of the spectrometer, and for which the virtual sources are represented at $S^{1}$ and $S^{2}$.

If the azimuth, $\vartheta_{D}$, of the asymmetry $O C=O C^{\prime}$ (of length $e_{D}$ ), is again referred to the bisector of the prism, as in figure 3 , part $1(b)$ of section III, then the lengths of the components perpendicular ${ }^{34}$ to the emergent rays are again $e_{D} \cos \left(D / 2+\vartheta_{D}\right)$ and $e_{D} \cos \left(D / 2-\vartheta_{D}\right)$, respectively, for the left- and right-hand deviations. Similarly for the incident rays the values are the same but their order is reversed.

${ }^{34}$ Since the angles $\alpha$ and $\beta$ are of the order of a few seconds in magnitude, all of the various incident and emergent rays may for this purpose be considered as parallel. 
The prism is approximately equidistant $\left(O S=O S_{1}=C S_{L}\right.$, etc. $)$ from the source $S$ and all of the virtual sources, the object distance of the source from the prism being

$$
-u_{p}=-v_{c}-x=\frac{f_{c}^{2}-f_{c} \Delta F_{c}}{\Delta F_{c}}-x
$$

where $v_{c}$ represents the distance from the collimator objective to the image of the source, and $x$ is the distance from the collimator objective to the prism table axis. The term $x$ can be ignored for the small values of $\Delta F_{c}$ which are considered, and likewise the term $-f_{c} \Delta F_{c}$ may be omitted as small in comparison with $f_{c}^{2}$. Then the equations

and

$$
\begin{aligned}
& \alpha_{1}=\frac{\Delta F_{c} e_{D} \cos \left(\frac{D}{2}+\vartheta_{D}\right)}{f_{c}^{2}} \\
& \alpha_{2}=\frac{\Delta F_{c} e_{D} \cos \left(\frac{D}{2}-\vartheta_{D}\right)}{f^{2}{ }_{c}} \\
& \beta_{1}=\frac{\Delta F_{c} e_{D} \cos \left(\frac{D}{2}-\vartheta_{D}\right)}{f^{2}{ }_{c}} \\
& \beta_{2}=\frac{\Delta F_{c} e_{D} \cos \left(\frac{D}{2}+\vartheta_{D}\right)}{f^{2}{ }_{c}}
\end{aligned}
$$

specify the values of the various angles $\alpha$ and $\beta$.

These values (39) when substituted in equation (37) reduce the latter to

$$
\Delta D=0
$$

a result which shows that, to a first approximation, no care in collimation of the incident light is necessary because of the asymmetric tabling of a (plane-surface) prism, provided the double minimum deviation is measured. Incidentally, this is an important reason for the measurement of $2 D$ even under circumstances where it is possible to make precise settings on the direct undeviated slit image.

\section{EFFECT OF ECCENTRIC TABLE AXIS WHEN INCIDENT LIGHT IS UNCOLLIMATED (FLAT PRISM SURFACES)}

As in part 2, section III, the special assumption (6') relating to coincident axes must again be supplanted by $\left(6^{\prime \prime}\right)$ which specifies correct tabling with respect to the axis of the table. Figure 8 and the notation illustrative of this case are already familiar from the preceding discussions and full details are unnecessary. The correct double deviation, $2 D$, is $\angle T_{1} O T_{2}$ and also, since no change in orientation of incident light occurs between pointings, $2 D=\angle t_{L} C t_{R}$. The measured double deviation, $2 D_{\epsilon}$, is $\angle T_{L} O T_{R}$, and the angles $t_{R} S_{R} T_{R}$ and $T_{L} S_{L} t_{L}$ will be designated as $\gamma$ and $\delta$, respectively. Then from figure 8

$$
2 D_{\epsilon}=2 D+\gamma+\delta
$$


and hence

$$
\Delta D=\frac{\gamma+\delta}{2}
$$

is the error in minimum-deviation measurement.

The orientation, $\varphi$, of the table-axis eccentricity, $O C$ is of course constant for both left and right deviations, and the lengths of the

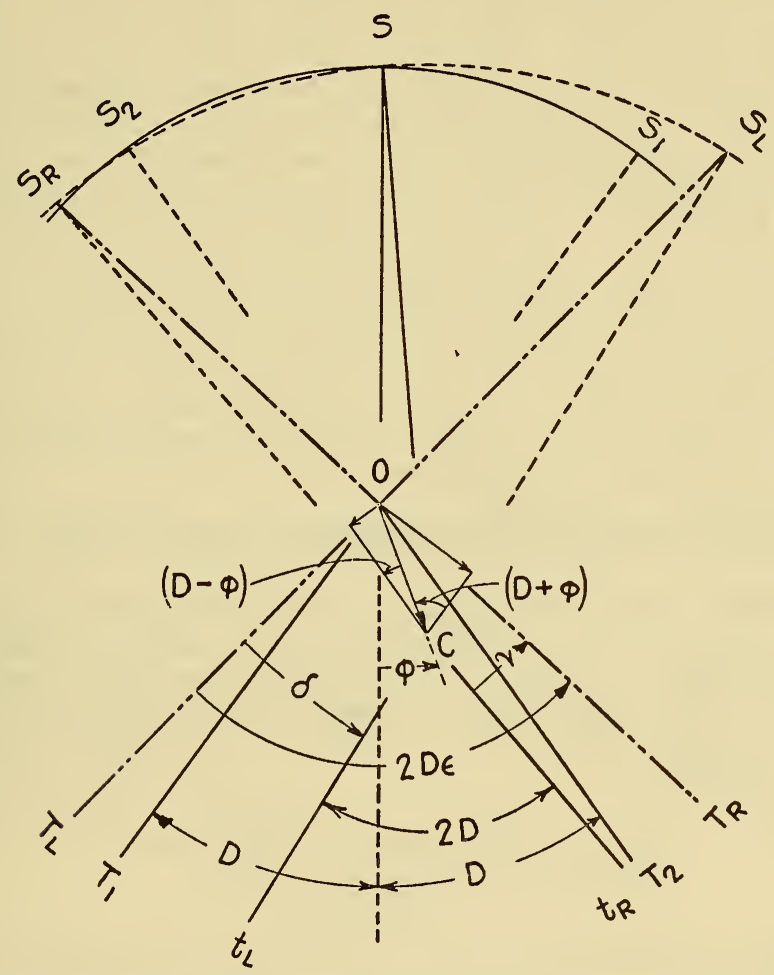

FIGURE 8.-Minimum-deviation measurement of a prism symmetrically tabled with respect to an eccentric prism-table axis when using uncollimated incident light.

As in figure 5 the chief ray intersections are again coincident with the eccentric prism-table axis at $C$, the prism outlines being omitted. In practice the eccentricity, $\epsilon=O C$, is small in proportion to the dimensions of the prism; the distance to the source, $S$, is very large; the virtual sources are sensibly coincident with their positions for an eccentricity of zero; and the angles $\gamma$ and $\delta$ do not exceed a few seconds in magnitude. The resulting error in the measured deviation becomes zero when $\epsilon$ has an aximuth, $\varphi$, of $\pm 90^{\circ}$.

components of $O C$ perpendicular to the emergent rays, left and right, respectively, are $\epsilon \sin (D+\varphi)$ and $\epsilon \sin (D-\varphi)$. With these lengths and equation (38) the error in deviation as given in equation (42) becomes

$$
\Delta D=\frac{\Delta F_{c} \epsilon \sin D \cos \varphi}{f_{c}^{2}}
$$

where, as previously in this section, $\Delta F_{c}$ is positive for a decrease in collimator tube length. 
(a) EXPERIMENTAL DETERMINATION AND AZIMUTHAL ADJUSTMENT OF ECCENTRICITY OF TABLE AXIS

If, for a known comparatively large value of $\Delta F_{\mathrm{c}}, \Delta D$ is experimentally determined for a few orientations of the prism table support, using a prism with plane surface, equation (43) may then be used to determine $\varphi$ and $\epsilon$. Obviously the instrument should finally be adjusted so that $\varphi= \pm 90^{\circ}$. If this adjustment can be made it seems that table-axis eccentricity can produce no first order errors in refractive-index measurements even when collimation is somewhat inaccurate.

By using equation (43) in this way, the writer computed a value of $\epsilon=0.16 \mathrm{~mm}$ for a spectrometer which he was using and then the prismtable support was oriented so that $\varphi$ (as simultaneously computed) became approximately $+90^{\circ}$. Later, a small pin was mounted on the prism table and a wire was mounted on the telescope in such manner that its bent and sharpened point hung just above the pin point. Then, while observing with a suitably mounted microscope of low power, the pin and wire points were adjusted so that no translation occurred as prism table and telescope were rotated through $180^{\circ}$. In fair agreement with the computations, the approximate values $\epsilon=0.2 \mathrm{~mm}$ and $\varphi=+100^{\circ}$ were then directly determined from the relative positions of the points.

(b) TOLERANCE IN COLLIMATION ADJUSTMENT CORRESPONDING TO A TABLE. AXIS ECCENTRICITY OF $0.2 \mathrm{MM}$

Nevertheless, since these values, $\varphi$ and $\epsilon$, are usually unknown, it is useful to determine the accuracy in collimation of incident light which is necessary in order to insure that index errors are negligible even when a fairly high value of $\epsilon$ exists at an azimuth $\varphi=0$ or $180^{\circ}$. Consequently equations (16) and (43) have been used in expressing this error in index

$$
\Delta n_{\Delta F_{c}}=\frac{\Delta F_{c} \in \sin D \cos \frac{A+D}{2} \cos \varphi}{2 f^{2}{ }_{c} \sin \frac{A}{2}}
$$

and therefore

$$
T_{\Delta F_{c}}= \pm \frac{2 \times 10^{-6} f^{2}{ }_{c} \sin \frac{A}{2}}{\epsilon \cos \varphi \sin D \cos \frac{A+D}{2}}
$$

is the corresponding tolerance in collimation adjustment. Figure 9 (a) gives values ${ }^{35}$ of $T_{\triangle F c} / f^{2}$ for $\cos \varphi= \pm 1$, and $\epsilon=0.2 \mathrm{~mm}$ (the latter being the maximum value suggested in sec. III, pt. $2(d)$, as generally permissible). For a collimator with any particular $f_{c}$ (measured in millimeters), the permissible error in tube length is readily found by applying the factor $f^{2}{ }_{c}$ to the values given just

${ }^{35} \mathrm{By}$ taking with respect to $n$ the partial derivative of the tolerance in inaccuracy of collimation (equation (45), expressed in terms of the variables $A$ and $n$ ) the most favorable condition is found to be

$$
2 \cot D=\tan \frac{A+D}{2}
$$

and this is expressed in fig. 9 by an undesignated dashed line. 
above these curves. For the special case, $f_{c}=400 \mathrm{~mm}$, the curves as drawn in figure $9(a)$ become contours of $\pm \Delta F_{400}=1.0,1.4,1.9,2.4$, and $2.9 \mathrm{~mm}$.

From these results it is evident that the values of table-axis eccentricity which are otherwise allowable or likely to occur on an accurate goniometer, do not necessarily impose severe tolerances in collimation adjustment when measuring refractive indices even if $\varphi$ is unknown and may, therefore, have one of the unfavorable values, 0 or $\pi$. It should be remembered, however, that this tolerance varies directly as the square of the collimator focal length. This suggests a possible reason for not limiting the focal lengths of precision spectrometer collimators to the comparatively small dimensions which would be quite adequate ${ }^{36}$ in the matter of requisite metrological accuracy.

In order to use a constant collimator tube length for all wave lengths of the visible spectrum, the collimator should be focused at

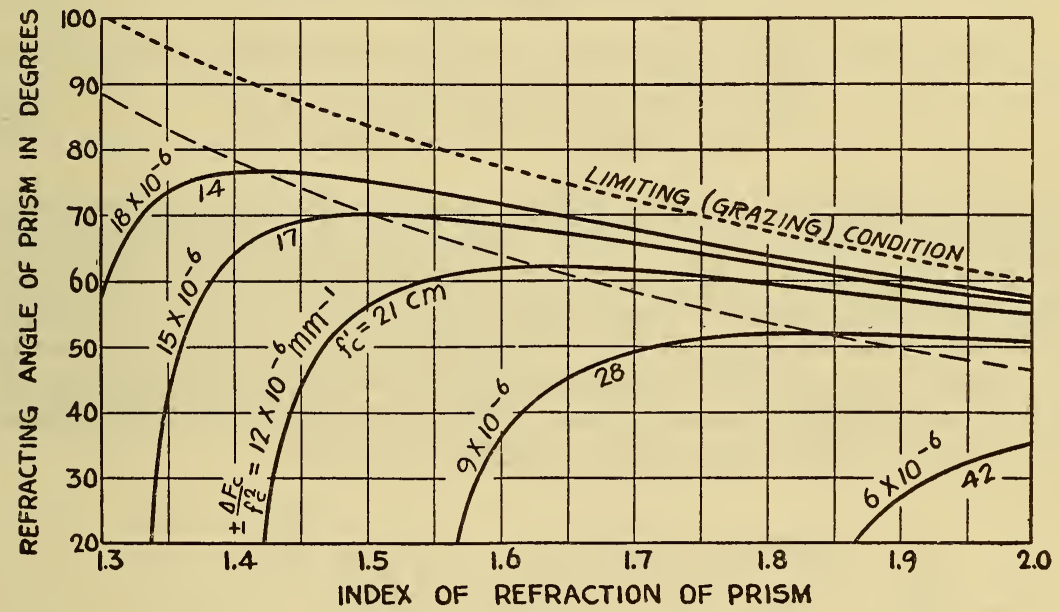

FIGURE 9

(a) Tolerance contours for adjustment of collimator tube length.-If unfavorably oriented table-axis eccentricity does not exceed $0.2 \mathrm{~mm}$, then any collimator may be inaccurately focused to the extent shown by these contours (read designations just above the curves and multiply by $\left(f^{2} c\right)$.

(b) Contours for minimum $f_{c}$ consistent with no chromatic refocusing of an achromatic collimator.-In the visible region a constant collimator tube length is usable if its focal length is at least as large as the values shown just below these same curves.

the middle of the linear range of required tube lengths. An adequate value of $f_{c}$ for this purpose is given by the equation ${f^{\prime}}_{c} \equiv 0.0025$ $\left(\Delta F_{c} / f_{c}^{2}\right)$, where the values of $\left(\Delta F_{c} / f_{c}^{2}\right)$ are those computed by equation (45) or read from the curves of figure $9(a)$ and 0.0025 is taken as one half the total range of longitudinal chromatic aberration for an achromatic objective of unit focal length. Accordingly the curves of figure 9 also serve $(b)$ as contours of adequate collimator focal length (read designations just below curves).

\section{RELATION OF PRISM ABERRATION TO COLLIMATION AND TO CURVATURE OF SURFACES}

A matter to be investigated before a final decision regarding the requisite precision in adjusting collimator tube length and the limit-

${ }^{36}$ See p. 76 of second paper cited in footnote 1, p. 26. 
ing permissible values of prism-surface curvature, is that of the aberration introduced by the prism. Does the whole beam or pencil remain sensibly symmetrical about the chief ray after refraction by the prism, or is sufficient asymmetrical aberration introduced to vitiate the accuracy of deviation measurements? Wadsworth ${ }^{37}$ examined the special case of a prism with plane surfaces and, corresponding to $\lambda / 16$ as the limiting permissible relative retardation, he found a very liberal tolerance in the requirements for collimator focusing. It is however not at once apparent that the effects of prism surface curvature can be ignored, and a more general case will now be considered.

For a homogeneous prism of negligible thickness, oriented for minimum deviation, Rayleigh ${ }^{38}$ gives, for rays in the primary plane, an equivalent of the equation

$$
\begin{gathered}
\frac{c^{2}}{v}=\frac{c^{2}}{u}+\left(n c^{\prime}-c\right)\left(\frac{1}{r_{1}}-\frac{1}{r_{2}}\right)+\frac{3 y^{\prime} \sin \frac{A+D}{2}\left(n^{\prime}-1\right)}{2}\left\{2\left(n^{\prime}+1\right)\left(\frac{c^{\prime}}{u^{\prime}}\right)^{2}\right. \\
\left.-\left(2 n^{\prime}+1\right)\left(\frac{1}{r_{1}}+\frac{1}{r_{2}}\right) \frac{c^{\prime}}{u^{\prime}}+n^{\prime}\left(\frac{1}{r_{1}^{2}}+\frac{1}{r_{2}^{2}}\right)\right\}
\end{gathered}
$$

where, in addition to the notation used in the similar first-orderimagery equation (10), $u^{\prime}$ is the image distance after refraction at the first surface, $y^{\prime} \equiv L / 2$ is the semiwidth of effective aperture as measured along the first face of the prism, and $n^{\prime} \equiv n c^{\prime} / c$. If there is imposed the condition $r_{2}=-r_{1}$ (the one which in practice must be approximately satisfied if prisms with but two polished surfaces are to be used for index measurement) then by comparison of equations (10) and (46) the longitudinal aberration reduces to

$$
\Delta v=-\frac{3 y v^{2} \sin \left(\frac{A+D}{2}\right)}{c^{3}}\left\{\left(n^{\prime 2}-1\right)\left(\frac{c^{\prime}}{u^{\prime}}\right)^{2}+\frac{n^{\prime}\left(n^{\prime}-1\right)}{r^{2}}\right\}
$$

where $y=y^{\prime} c$ is the semiwidth of the (cross-sectional) aperture of the incident pencil at the first face of the prism.

By neglecting the aberration itself in the equation which expresses the refraction at the first face of the prism and by replacing $u$ with one term of equation (38), $u^{\prime}$ is expressible approximately as

$$
u^{\prime}=-\frac{f^{2}{ }_{c} r n^{\prime} c^{\prime}}{c r \Delta F_{c}-f^{2}{ }_{c}\left(n^{\prime}-1\right)}
$$

and instead of equation (47) one may then write

$$
\Delta v=-\frac{3 y n v^{2} \sin \frac{A}{2}}{c^{3}}\left\{\frac{\left(n^{\prime 2}-1\right)\left[r c \Delta F_{c}-f_{c}^{2}\left(n^{\prime}-1\right)\right]^{2}}{f^{4}{ }_{c} r^{2} n^{\prime 2}}+\frac{n^{\prime}\left(n^{\prime}-1\right)}{r^{2}}\right\}
$$

to express the longitudinal aberration.

37 F. L. O. Wadsworth, Astrophys. J., vol. 17, pp. 9-11, 1903.

${ }_{38}$ Lord Rayleigh, Phil. Mag. (5), vol. 9, pp. 46-47, 1880. A change in signs has been made to conform to the convention that distances measured from the vertices of refracting surfaces are positive in the direction of travel of the light. 
To find the corresponding restrictions imposed upon refocusings of the collimator it is convenient to use the equation of transformation from longitudinal aberration to phase difference in the form

$$
\eta=\frac{1}{\lambda v^{2}} \int_{0}^{y} \Delta v y d y
$$

similar to that given by Martin, ${ }^{39}$ where $\eta$ is the phase difference in periods or cycles. After substituting equation (49) in (50) and adopting the Wadsworth value of $|\eta|=1 / 16$ period as the permissible limit of phase difference the corresponding particular limits in refocusing of the collimator are readily established as

$$
\Delta F_{c}= \pm \frac{n c^{\prime} f_{c}^{2}}{c} \sqrt{\frac{\lambda}{2 L^{3} n\left(n^{2}-1\right) \sin (A / 2)}-\frac{n c^{\prime}}{r^{2} c^{2}\left(n c^{\prime}+c\right)}}+\frac{f_{c}^{2}\left(n c^{\prime}-c\right)}{r c^{2}}
$$

where $2 y$ has been replaced by $L c$, the projection of $2 y$ along the prism face, and where, for sixth decimal place refractometry, $L$ need not exceed $\operatorname{cosec} \frac{A}{2}$ centimeters. ${ }^{40}$ The successful use of such small prisms depends on realizing something closely approximating optimum metrological power and thus, to the extent ${ }^{41}$ that $\lambda / 16$ approximates the corresponding limit in relative retardation, equation (51) establishes, at least from the standpoint of prism aberration, a safe limit for inaccuracy of collimation when seeking a precision of $\pm 1 \times 10^{-6}$ in a measurement of refractive index.

For a prism with plane surfaces, $r=\propto$, equation (51) reduces to

$$
\Delta F_{c}= \pm \frac{n c^{\prime} f_{c}^{2}}{L c} \sqrt{\frac{\lambda}{2 \operatorname{Ln}\left(n^{2}-1\right) \sin A / 2}}
$$

in exact agreement with the above-mentioned result by Wadsworth. Furthermore, by making $\Delta F_{c}=0$ in (51), to correspond to the special case of curved surfaces and collimated light, one may solve for $1 / r$ and obtain

$$
1 / r= \pm \frac{n c c^{\prime}}{L} \sqrt{\frac{\lambda}{2 \operatorname{Ln}\left(n^{2}-1\right) \sin (A / 2)} \cdot \frac{\left(n c^{\prime}+c\right)}{\left(n c^{\prime}-c\right)^{2}\left(n c^{\prime}+c\right)+n^{3} c^{\prime 3}}}
$$

which are the particular limits in prism surface curvature within which the relative retardation for collimated light does not exceed $\lambda / 16$.

${ }^{39}$ L. C. Martin, Trans. Optical Soc., London, vol. 23, p. 66, 1921-22.

40 See p. 76 of second paper cited in footnote 1, p. 26.

41 For telescopic instruments Wadsworth concluded (Astrophys. J., vol. 16, pp. 270, 279, 1902) that 1/15 was a fair value for the ratio of the limit of metrological precision to that of resolution and then he used this ratio in making his estimate of $\lambda / 16$ as the permissible limit of relative retardation consistent with optimum accuracy of measurement. It should now be mentioned that, optically, the writer (see p. 64 of second paper cited in footnote 1, p. 26.) has found it easy to obtain values as small as $1 / 25$ or $1 / 30$ for the abovementioned ratio between metrological and resolution limits and that the corresponding limit of relative retardation is only about $\lambda / 32$. The necessity for such freedom from aberration may be questioned because of mechanical and, perhaps, other considerations, but nevertheless it is interesting to note that the use of this very high standard would simply replace 2 by 4 in the denominator of the first term under the radical in equation (51). The term over $r^{2}$ is negligible and, accordingly, this change would decrease the values of $\Delta F_{c}$, as listed in table 1 , by approximately 30 percent but would not affect the conclusions which are drawn therefrom. 
Numerical data on the prism-aberration tolerance in collimation, equation (51), are given in table 1 . The values of $T_{\Delta F_{c}} / f_{c}^{2}$, as adopted for the curves of figure $9(a)$, have again been computed and one wave length, $\lambda=5,461 \mathrm{~A}$, is adequate in showing the order of magnitude of this tolerance. For $r$ the positive values according to equation (23) have been read from figure $4(a)$. The results given in table 1 do not differ by as much as 5 percent from similar results computed for plane-surface prisms from equation (52). It is evident that this prism-aberration tolerance in collimation is very large in comparison with that which has been considered in figure $9(a)$ as a result of possible table-axis eccentricity. Moreover, equation (53) shows that prism aberration is not a factor which compels reconsideration of those tolerances in prism-surface curvature which are based on an easily attainable degree of precision in the tabling of prisms and according to equation (23) are expressed in figure $4(a)$.

TABLE 1.-Prism-aberration limits for inaccuracy of collimation

[Values of $\frac{\Delta F_{c}}{f_{c}{ }^{2}} \times 10^{5}$ computed from equation (51)]

\begin{tabular}{|c|c|c|c|c|}
\hline & $n=1.3$ & $n=1.5$ & $n=1.7$ & $n=1.9$ \\
\hline$A=80^{\circ}$ & $\left\{\begin{array}{l}+66 \\
-63\end{array}\right.$ & $\begin{array}{l}+110 \\
-104\end{array}$ & & \\
\hline$A=60^{\circ}$ & $\left\{\begin{array}{l}+42 \\
-40\end{array}\right.$ & $\begin{array}{r}+38 \\
-37\end{array}$ & $\begin{array}{l}+42 \\
-40\end{array}$ & $\begin{array}{l}+64 \\
-60\end{array}$ \\
\hline$A=40^{\circ}$ & $\left\{\begin{array}{l}+27 \\
-26\end{array}\right.$ & $\begin{array}{l}+22 \\
-21\end{array}$ & $\begin{array}{l}+20 \\
-19\end{array}$ & $\begin{array}{l}+20 \\
-19\end{array}$ \\
\hline
\end{tabular}

Note.-By comparing the values of this table with the contours of figure $9(a)$ it is found that these permissible inaccuracies of collimation exceed by at least one order of magnitude those tolerances which are based on table-axis eccentricity.

\section{SUMMARY AND DISCUSSION}

The necessity of flat prism surfaces has been greatly emphasized in prism refractometry, and accurate collimation has been generally considered of major importance. Perhaps this is mainly because the split-beam method of angle measurement, which was formerly in use by many of the most careful observers, involves the inherent weaknesses of asymmetry which make it impracticable for accurate work even with the best surfaces which can be realized and with optimum adjustment of the collimator. Under such circumstances it is not surprising that undue stress has been placed on the importance of accurate collimation and that it has been considered impossible to make accurate refractive index measurements on prisms having curved surfaces.

Starting with the imperative necessity of using all lens and prism apertures symmetrically, even under the most favorable conditions, it is then found that a strict observance of this principle permits useful tolerances in curvature of prism surfaces and in collimation adjustment. The most exacting requirement in the case of prism surfaces is that the curvatures on a given prism must be equal to the extent that a satisfactory compromise focus of the autocollimating telescope can be realized for making refracting-angle measurements between planes tangent to the effective prism-surface centers. The magnitude of curvature which can be permitted is limited by the precision with 
which prisms can be translated on the table of the spectrometer. When many prisms are required for refractive-index measurements a specification for approximately equi-curvature of not exceeding, say, $\lambda / 3$ for $1 \mathrm{~cm}$ diameter of surface greatly facilitates their preparation as compared with a specification for say $\lambda / 20$.

Accurate collimation is not required because of asymmetric tabling, provided the "double deviation" is observed, and there are no important limitations on collimator refocusing because of the aberration of prisms having surfaces with curvatures which are otherwise permissible. Tolerances in collimation may, however, be limited by eccentricity of the prism-table axis. Nevertheless, if the latter does not exceed $0.2 \mathrm{~mm}$ (or if its azimuth can be favorably oriented) then all wave lengths of the visible spectrum may be used for index measurements on $60^{\circ}$ prisms with a constant collimator tube length (not less than $22 \mathrm{~cm}$ ), and all of the refocusings be easily and quickly made with the telescope. In making large numbers of high-precision index measurements, this new observational procedure which eliminates the customary and troublesome collimation adjustments is a time-saving feature of self-evident value.

For such procedure the collimator should be initially adjusted to a mean between the extremes of the various focal lengths corresponding to the different wave lengths which are to be used. Obviously, the necessary range of refocusing of the telescope is twice the longitudinal chromatic aberration of either of the (identical) objectives for the spectral region which is concerned but the resulting linear error in focusing the collimator for parallel light never exceeds one half this color focal difference. For objectives of the usual two-color-correction type, the total range of longitudinal chromatic aberration for wave lengths of the visible spectrum is of the order of $0.005 f$ or less. Figure 9 (a) shows that, with $f=400 \mathrm{~mm}$ and $A=30^{\circ}$ or more, the tolerance in collimator refocusing is at least as great as \pm 1 or $2 \mathrm{~mm}$, provided $\epsilon$ does not exceed $0.2 \mathrm{~mm}$, and these ranges are one or two times as large as the requisite one half of the chromatic variation in focal length. Usually there is ample provision for larger changes in $F_{c}$ or for larger values of $\epsilon$, and thus even from this consideration of convenience a collimator longer than $40 \mathrm{~cm}$ seems unnecessary for minimum-deviation measurements unless it is desired to limit individual errors to something less than approximately $\pm 1 \times 10^{-6}$ in the computed index of refraction.

Washington, March 29, 1933. 